\title{
Multicomponent Mass Transfer Kinetics, Concentration Waves in the Diffusion Model for Nonselective Ion Exchange
}

\author{
Anatoliy Kalinitchev \\ Institute for Phys. Chem. \& ElectroChem., Rus. Academia of Science, GSP, Leninsky Prospect, 31, 119991 Moscow, Russia \\ *Corresponding Author: kalin_phyche@mail.ru
}

Copyright (C) 2013 Horizon Research Publishing All rights reserved.

\begin{abstract}
The aim of this theoretical investigation is to describe the $i$-component concentration waves behavior inside the nonselective ion exchanger matrix by using the modern Model for the multicomponent mass transfer kinetics. The author's generalized theoretical Model for the detailed description of the properties of the bi-functional matrixes with the two functions: I-Selectivity \& II Diffusivity ( $D_{i}$ for $i$-components) was proposed previously. This new generalized Model is assigned for the investigations of the multi-component mass transfer kinetics in the novel bi-functional materials including NanoComposites (NC). The partial case of the systems for the multicomponent diffusion kinetics in the ion exchangers without Selectivity factor (I) influence is considered here. For the such nonselective Ion Exchange (IEx) systems it is possible to investigate only one factor influence (II, Diffusion) with the $D_{i}$-Diffusivities of $i$-components, in other words the partial case of the generalized Model mentioned. The results of the computer simulation of the ternary IEx system (A,B,C - ionic components) demonstrate the availability of the noval displacement effect during the propagation and interference of the two (fast and slow) diffusion $B, C$-concentration waves for the two kinds of $B, C$-ions introducing into the IEx matrix from the outer solution. It is shown that the $B$-accumulation occurs due to the interference of the $B, C$-waves. Computerized modeling for the two various shape of the resin: r-bead and ro-fiber shows that the new displacement effect is more appreciable for the $\mathbf{r}$-bead of the ion exchangers. The explanations of the displacement effect including values of the diffusivities - $D_{A}$, $D_{B}, D_{C}$ are given.
\end{abstract}

Keywords Mass Transfer, Concentration Waves, Nano-Composites, Diffusion, Multi-Component Kinetics, IEx Matrix, Fixed Groups, New Displacement Effect

\section{Introduction}

There was proposed previously the modern multicomponent generalized kinetic Model created for the theoretical investigations of the mass transfer kinetics inside the bi-functional matrix with the key conception: two co-existing routes (I,II) for the multicomponent mass transfer inside the bi-functional matrix-medium [1-4]. These two co-routes reflect the two simultaneous processes: (I) selective sorption-desorption for the $i$-components at the active nano-sites (Selectivity factor) of the bi-functional matrix, and (II) - diffusion $\left(D_{i}\right)$ of the $i$-components mixture $\mathrm{n}$ the pores (Diffusivity factor) of the same matrix. As the additional illustration of the main postulates the simple scheme of the mass transfer in the generalized Model for the bi-functional matrix is presented also [1-4].

This generalized Model approach with the bi-functional matrix includes such popular high-tech materials as NanoComposites (NC). The NC include a lot of the NanoParticles (NP) embedded in advance inside the matrix-medium of the NC. With the use of the additional very important concentration wave approach $\left(\mathrm{W}^{+}\right)$in the such modern Model it was shown that the influence of the two factors (I,II) brings the combined influence of them on the rate of the kinetic process. Besides, the two (I,II)-factors affect the interference of the concentration waves-profiles inside the various shape of the bi-functional medium: $\mathbf{r}$-bead, ro-fiber and L-membrane [1-4].

Then the computerized generalized Model was used for the theoretical investigation of the multicomponent mass transfer intra-particle diffusion kinetics including association-dissociation process in the NC. In result the conclusions were deduced that the effect of this combined action of the both factors: I (Selectivity), and II (Diffusivity) is completely similar to the combined Equlibrium \& Diffusion (HETP) factors influence in the theory of chromatography [1-4]. The additional very important key "wave concept" - $\mathrm{W}^{+}$plays the main role in the Model mentioned.

There is one important partial case for the generalized bi-functional Model when the influence of only one second 
diffusion factor II (Diffusivity, $D_{i}$ ) may be taken into the consideration. For the simple nonselective ion exchange (IEx) the influence of the first factor I (the Selectivity) may be reasonably neglected. Consequently the factor $\mathbf{I}$ of the described Model may be excluded from the consideration. The intra-particle multicomponent nonselective IEx includes the influence of only factor II (Diffusion): the diffusion of the $i$-components-ions (with the $D_{i}$ diffusivities) during the IEx kinetics. Here such diffusion ternary IEx kinetic process - $\left(B^{+}+C^{+}\right) / \mathrm{R} A$ is considered in the mono-functional matrix as the partial case of the generalized Model. It gives the possibility to estimate separately the influence of only one multicomponent factor (II) with the $D_{i}$-diffusivities $(i=A, B, C)$ on the rate characteristics of the ternary $\left(A^{+}, B^{+}, C^{+}\right)$ IEx kinetics. Naturally that the key concept of the concentration wave $\left(\mathrm{W}^{+}\right)$of the Model plays the same important role for the partial case considered here. It is important that the Model gives the possibility to estimate the propagation and the interference-interaction of the $A, B, C$-concentration waves in the nonselective IEx matrix.

In the Figures $1(\boldsymbol{a}, \boldsymbol{b})$ it is shown as the illustration - the experimental example of the simple one component propagating $i$-concentration wave-profile inside the IEx matrix of the VPC resin for the two types of the binary IEx: (a) $\mathrm{Ni}^{+} / \mathrm{H}^{+}$, and (b) $\mathrm{Ni}^{+} / \mathrm{Na}^{+}$. Therewith, the experimental micro-picture (Fig. 1b) presents the visual illustration of the IEx medium structure:

There are described a lot of the theoretical investigations for the multicomponent IEx kinetics including various IEx Models in the well known monograph [5] with taking into account the reviews [6,7]. The kinetic intra-particle mass transfer in the r-beads for the ternary IEx: $\mathrm{RA}_{\text {resin }} /(\mathrm{B}+\mathrm{A})_{\text {solution }}$ has been investigated in a number of the various IEx studies [8,9] with the analyses of the kinetic curves $\mathrm{F}_{i}(\mathrm{~T}) \quad(i=A, B, C)$. However the concentration multicomponent $i$-waves behavior in the ion exchangers has not been studied in the articles [6-8]. For the selective ternary IEx the theoretical studies of the $i$-concentration waves behavior inside the IEx matrix has been fulfilled on the basis of the author's approach [9]. A number of the additional investigations in the IEx kinetics are cited in the previous author's articles [1-4].

(a).

\section{VPC ampholyte resin bead}

\section{(a) $\mathrm{Ni}^{+} / \mathrm{RH}$}

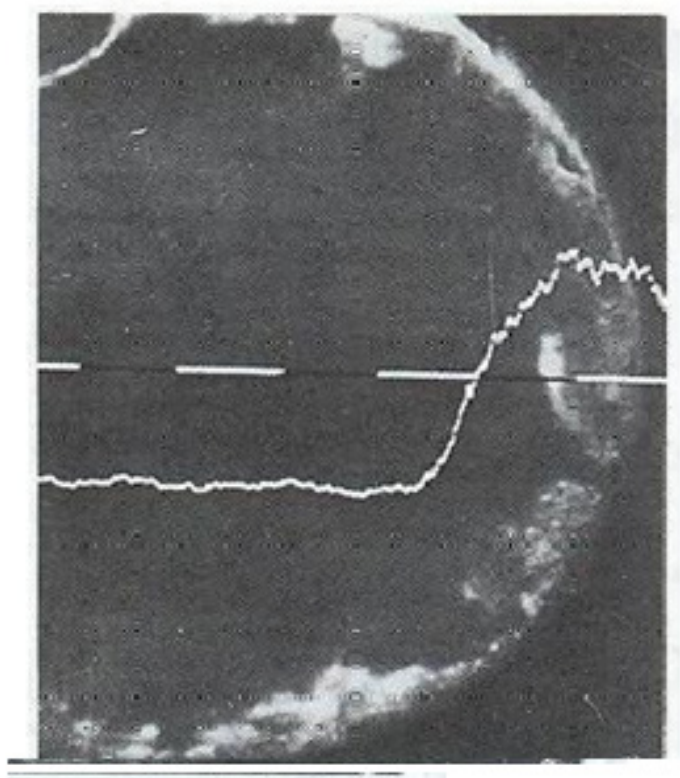

(b) $\mathrm{Ni}^{+} / \mathrm{RNa}$

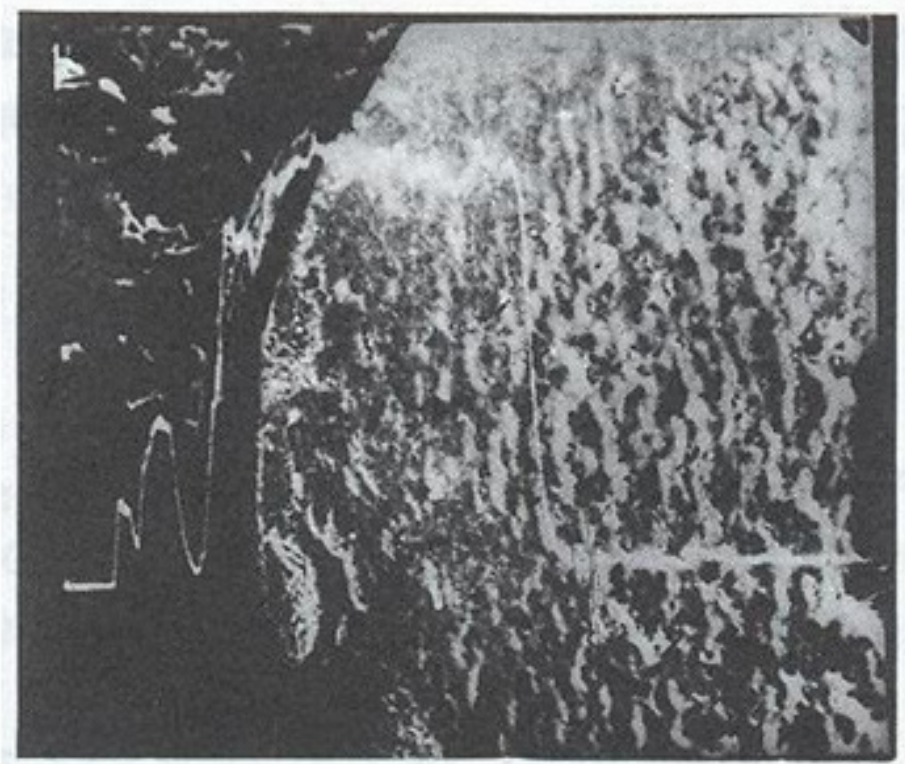

Figure 1 $(\boldsymbol{a}, \boldsymbol{b})$. Experimental SCM micro-pictures with one component concentration waves inside r-bead of the VPC resin (Russia). Light lines show the concentration waves - profiles for two kinds of the binary IEx: $(\boldsymbol{a}) \mathrm{Ni}^{+} / \mathrm{RH}$ - micro-picture of the spreading wave to the center of resin bead of nonselective IEx; (b) $\mathrm{Ni}^{+} / \mathrm{RNa}$ - micro-picture of the sharp wave propagating to the center of resin bead for the selective IEx [9]. 
For this theoretical study of the multicomponent IEx kinetics there is important to note the article [8] including the previously obtained experimental results with the integral kinetic curves $\mathrm{F}_{i}(\mathrm{~T})$, where it was obtained accumulation of the mean (integral) $\mathrm{B}$ - concentration for the $\mathrm{H}^{+}, \mathrm{Na}^{+}$ions. The kinetic experiments were done for the intra-particle ternary IEx systems (during sorption stage): $\left(\mathrm{Na}^{+}+\mathrm{Zn}^{2+}\right) / \mathrm{R} H^{+},\left(\mathrm{H}^{+}+\mathrm{Zn}^{2+}\right) / \mathrm{RNa}^{+}$for the various types of the IEx resins: Dowex\&Diaion [8]. The coupling effect of the electric field on the mass transfer is also included into the consideration via the Nernst-Plank relationship. It was found the experimental dependence of the $\mathrm{H}^{+}$ions accumulation (or $\mathrm{Na}^{+}$) presented as the moderate peak on the one kinetic curve $\mathrm{F}_{i}(\mathrm{~T})$ for the various diffusivities: $D_{\mathrm{H}}>D_{\mathrm{Na}}>>D_{\mathrm{Zn}}$. There was pointed out the following conclusion of the paper [8]: when the fastest ion $\mathrm{H}^{+}$(or ion $\mathrm{Na}^{+}$in other variant) was initially (at $\mathrm{t}=0$ ) in the resin phase the faster ion (from the two invading $A^{+}, B^{+}$- ions into the $\mathbf{r}$-bead) showed a peak in the one kinetic curve $\mathrm{F}_{i}(\mathrm{~T})$ but not in the case when the slowest ion, $Z^{2+}$ was in the resin phase. The gradient of the potential of the electric field, which influences the IEx kinetic process was included [8].

The non-monotonous behavior of the kinetic curve $\mathrm{F}_{i}(\mathrm{t})$ with the $\mathrm{F}_{i}{ }^{\max }$-peak was obtained experimentally in the membranes for the specific (impulse) boundary conditions including the electric field effects on the multicomponent IEx kinetics $[10,11]$. The intensive experiments with the IEx membranes were supported by the calculations on the basis of the macroscopic multicomponent IEx kinetic model [11]. However the authors $[8,10,11]$ have no possibility in principle to consider the $A, B, C$-concentration wavesprofiles behavior inside the matrix of the ion exchangers.

In all theoretical works it is usually used the mathematical approach with the application of the classical, and fundamental Nernst-Plank relationships for the $A, B, C$-ions fluxes $\left(\mathrm{J}_{i}\right)$ for the multicomponent mass transfer in the IEx systems. This approach is based on the principles of the non-equilibrium thermodynamics, which includes the coupled influences of the $i$-concentration gradients together with the gradient of the electric field in the IEx matrix. Then the application of the relationships for the electro-neutrality together with the absence of the electric current relation with including the Nernst-Plank relationships gives the mathematical exclusion of the gradient of the electric field from the consideration [1-9]. For the ternary IEx: $\left(\mathrm{B}^{+}+\mathrm{C}^{+}\right) / \mathrm{RA}$ it is shown [7-9] that the influence of the electric field forces with including of the $i$-concentration gradients affects the kinetic rate of the IEx processes due to the coefficients of the $D_{i}$-diffusivities of the $A, B, C$-ions.

In result of the author's computerized simulation $[2,3]$ it was obtained the most interesting non-monotonous behavior of the $\mathrm{F}_{B}(\mathrm{~T})$ kinetic curves with the presence of the kinetic maximum $-\mathrm{F}_{B}{ }^{\max }$ for $B$-ions in the partial case of the ternary nonselective IEx kinetics for the some variants with the $D_{i}$ -diffusivities values $\left(D_{\mathrm{B}}>D_{\mathrm{A}}>D_{\mathrm{C}}\right)$. Such variants were calculated on the basis of the Model with some $D_{i}$ -diffusivities for the $\mathbf{r}$-bead [2,3]. Moreover, the results of the modeling include the calculations of the profiles of the $i$ concentration waves $(i=A, B, C)[2,3]$. Several variants of the IEx: $\left(B^{+}+C^{+}\right) /$RA were modeled for the three shapes of the IEx matrix: L-membrane, ro-fiber, $\mathbf{r}$-bead [2,3]. In the investigation here the study has been continued for the rather wide diapason of the $D_{i}$-diffusivities including the comparative consideration of the interference of the $i$-concentration diffusion waves for the multicomponent IEx kinetics inside the $\mathbf{r}$-bead, and ro-fiber. The results obtained here allow to make more precise conclusions, and bring definitely the new real reasons for the non-monotonic (with $\mathrm{F}_{B}{ }^{\max }$ maximum) behavior of the kinetic curves $\mathrm{F}_{B}(\mathrm{~T})$. It will be shown that the new "displacement effect" for the $B$-concentration wave, arising as the result of the all $i$-waves interference, plays the main role in the non-monotonous $\mathrm{F}_{B}(\mathrm{~T})$ behavior.

\section{Phenomenological Approach for Modeling of Multicomponent Mass Transfer for IEx Kinetics}

Herewith the general phenomenological approach of the non-equilibrium thermodynamics has been used for the multi-component mass transfer in the nonselective IEx matrix [2,3,5-9]. The postulates for the various IEx multicomponent Models for IEx kinetics have been used during long-time period (around 50-60 years) starting from early prof. F. Helfferich book [5] through his Review [6] and with emphasizing STATE-OF-THE-ART-REPORT [7]. There is enumerated here (in short) the list of mathematical approaches in the description of the IEx kinetics: mass balance partial differential equations, quasi homogeneous medium, isothermal process, electro-neutrality, fundamental Nernst-Planck relationship for fluxes, constant diffusion coefficients $\left(D_{i}\right)$ for $i$-component mixtures. As usual, the influence of the gradient of the electric field is expressed via the sum of the $j$-concentration gradients by using the absence of the electric current relationship. [2,3,5-9] In result the flux of each $i$ - ion-component is described by its own gradient with addition of the multicomponent superposition of the another $j$-concentrations gradients [2,3,5-9]. Such superposition is called "diffusion potential" in the theory of irreversible thermodynamics.

For the computerized simulation of the multicomponent IEx kinetics there was used the phenomenological approach on the basis of the system of the mass balance partial differential equations for $i$-components (1a), fundamental Nernst-Plank relations ( $1 \mathrm{~b}$ ) for the $i$-fluxes $\mathrm{J}_{i}$, and the relation (1c) for the gradient of the electric field $(\Phi)$ in the IEx matrix [2,3,5-9]:

mass balance equations,

$$
\delta \mathrm{X}_{i} / \delta \mathrm{t}=-\operatorname{div}\left(\mathrm{J}_{i}\right) i=1,2, \ldots \mathrm{n}
$$

Nernst-Plank (N-P) relations,

$$
\mathrm{J}_{i}=\mathrm{J}_{i}^{\mathrm{X}}+\mathrm{J}_{i}^{\mathrm{el}}=-D_{i}\left[\operatorname{grad} \mathrm{X}_{i}+(F / \mathrm{R} T) \operatorname{grad} \Phi\right](1 \mathrm{~b})
$$

Gradient of the Electric field potential $(\Phi)$, 


\section{$-(F / \mathrm{R} T) \operatorname{grad}(\Phi)=\operatorname{SUM}_{\mathrm{s}}\left(\mathrm{z}_{\mathrm{s}} D_{\mathrm{s}} \mathrm{X}_{\mathrm{s}}\right) / \mathrm{SUM}_{i}\left\{\mathrm{z}_{i}^{2} D_{i} \mathrm{X}_{i}\right\}(1 \mathrm{c})$}

The first term in the N-P relations for the fluxes $\mathrm{J}_{i}(1 \mathrm{~b})$ is the flux $\mathrm{J}_{i}{ }^{\mathrm{X}}$ determined by the gradient of the $i$-concentrations and the second term is the flux $\mathrm{J}_{i}^{\mathrm{el}}$ determined by the forces of the electric field (1c).

This n-component mass balance IEx system (1) is described by the well known mathematical approach, where the relation (1c) for the gradient of the electric field potential: $\operatorname{grad}(\Phi)$ has been derived by using of the electro-neutrality relationships, and the relation of the absence of the electric current including the Nernst-Plank relations [5-9]. The relationship for the $\mathrm{J}_{i}$ fluxes (1b) shows that the kinetic rate and the propagation of the $i$-concentration waves depend on the $i$-concentration gradients altogether with the forces of the electric field. In the final result (see $1 b, c)$ all the parameters of the multicomponent IEx kinetics are influenced by the $D_{i}$-diffusivities of the $i$-ions $(i=A, B, C)$.

Thus in accordance with the phenomenological approach, under the influence of the $i$-concentration gradients there are formed the propagating $i$-concentration waves in the IEx matrix (r-bead, and ro-fiber) during the ternary kinetic IEx process: $\left(\mathrm{B}^{+}+\mathrm{C}^{+}\right) / \mathrm{RA}$. During computerized modeling the corresponding constant initial and boundary conditions are used with the computerized calculation of the $i$-concentration waves-distributions together (and simultaneously) with the integral kinetic curves $\mathrm{F}_{i}(\mathrm{~T})(i=\mathrm{A}, \mathrm{B}, \mathrm{C})$.

In the ternary IEx kinetic process: $\left(\mathrm{B}^{+}+\mathrm{C}^{+}\right) / \mathrm{RA}$ the incoming $\mathrm{B}, \mathrm{C}$-waves propagate across the various IEx media (r-bead, and ro-fiber) from the boundary points $\left(\mathrm{ro}_{0}, \mathrm{r}_{0}=1\right)$ to the final "zero points" (ro, $\mathrm{r}=0$ ) correspondingly. The outgoing $A$-concentration wave propagates in the opposite direction: from the IEx matrix to the boundary point $\left(\mathrm{ro}_{0}, \mathrm{r}_{0}\right.$ $=1$ ), Figs. $2 \boldsymbol{a}, \boldsymbol{b}$. The kinetic processes are finished at the completion moment $\mathrm{T}=\mathrm{T}^{\text {fin }}$, when the kinetic curve $\mathrm{F}_{i}(\mathrm{~T})$ for the matrix filling comes to the final state -"plato" for the each $i$-component concentration (Figs. 2a,b, right).

A number of the author's computer program for the mathematical solution of the partial differential equations (1) of the Model has been composed. The results of the computerized modeling for the multicomponent $i$-concentration waves $-\mathrm{X}_{i}(\mathbf{r}, \mathbf{r o} ; \mathrm{T})$ are placed in the files, which are used then, in particular, for the creature of the author's animations of the mass transfer kinetics mentioned. The animations show visually the whole kinetic process in the course of T-time, the propagation of the broadening $i$-concentration waves: $\mathbf{X}_{\mathrm{ir}, \mathrm{ro}}(\mathrm{T})$. Such presentation of the results of the computerized simulation is extremely productive, as it gives the fundamental and clear basis for understanding of the multicomponent mass transfer IEx process in the course of time - $T$.

The modern approach with the all mentioned postulates and equations of the Model for the bi-functional matrix has been realized by the computer modeling [1-4]. As the partial case of the generalized Model the set of the corresponding computer FORTRAN author's programs has been composed for the simulation of the multi-component IEx diffusion mass transfer in the IEx systems, describing kinetic behavior inside the IEx mono-functional matrix. Thus there are realized the computerized investigations of the influence of the $D_{i}$-diffusivities of the $i$-components on the IEx diffusion mass transfer kinetics inside the resins: the r-beads, and ro-fibers.

Concentration waves arise at the start (when time, $\mathrm{T}^{1}=0$ ) of the process (Figs. $2 \boldsymbol{a}, \boldsymbol{b} ; \mathrm{T}_{\mathbf{r}}{ }^{1}, \mathrm{~T}_{\mathbf{r o}}{ }^{1}$ ), and then move along the $\mathbf{r}$-,ro-distances (radii) inside the matrix of the ion exchanger during the multicomponent mass transfer. The multicomponent $i$-concentration waves with their propagation by the diffusion in the IEx matrix play the decisive role in the description of the multicomponent diffusion IEx kinetics. Therefore this well known and widely used "wave" approach $[1-9,12-17]$ is shortly reviewed in the next Section 3.

\section{Concept of Concentration Waves $\left(\mathbf{W}^{+}\right)$ for Multicomponent Mass Transfer Kinetics}

During the mass transfer kinetics the $i$-concentration "waves"-profiles of the $i$-components are aroused inside the porous media. Figures $1(\boldsymbol{a}, \boldsymbol{b})$ above represent the simple experimental examples of the one component diffusion waves propagating during the multi-component mass transfer IEx kinetics. The key concept $\left(\mathrm{W}^{+}\right)$of "the multi-component $i$-concentration waves" is widely used in the theoretical description for the many scientific fields of the multi-component transport for various kinetic and dynamic systems. The "multi-component waves" concept $\left(\mathrm{W}^{+}\right)$has wide area for applications in such research fields, as theory of chromatography [12,15-17], percolation processes [15], mechanics of liquids, gas dynamics, theory of burning and even street traffic [15]. The extended literature is presented in the author's articles [1-4]. There are phenomenological concepts potentially common to the all filtration processes, which can also be extended to a whole series of the migration phenomena such as chromatography, sedimentation, electrophoresis and some others. [12-17] The term "wave" $\left(\mathrm{W}^{+}\right.$concept, here) has been used in all such publications [9,12-17] including especially the mentioned excellent monograph on the theory of multicomponent chromatography. [12]

Waves propagation, as well as their concurrent interference-interaction should be used for the simulation and consideration of all the details of the multicomponent mass transfer kinetics and dynamics [1-4,7-9,12-17]. The fundamental monograph [12] (including additionally a lot of the chromatographic papers by F. Helfferich [13]) is devoted to the all aspects of the travelling "concentration waves" concept including the waves interference. There is a lot of the research papers adjoined, which are included into the special issue [13] dedicated to prof. F. Helfferich (Festschrift) with the detailed consideration of the "propagating multi-component concentration waves" 
concept [12-17], and some others related [1]. In the presence of the effects of the non-ideality of the sorption medium the travelling concentration waves propagate with their broadening, including effects of their consequent interference. [12-17]

The concept of the "multi-component concentration waves" $\left(\mathrm{W}^{+}\right)$is widely used here to consider the results of the computer simulation of the multi-component IEx kinetics in the mono-functional IEx matrix. The examples of the multi-component propagating $i$-concentration waves are presented in the series of the pictures in Figs. $2 \boldsymbol{a}, \boldsymbol{b}$. Figures $2 \boldsymbol{a}, \boldsymbol{b}$ show the $i$-concentration waves in the IEx matrix: from the $\mathrm{X}_{i}\left(\mathbf{r}, \mathbf{r o} ; \mathrm{T}^{\mathrm{k}}\right)$-profiles $(l e f t)$ to the kinetic $\mathrm{F}_{i}(\mathrm{~T})$-curves $(r i g h t)$ in course of T-time. In such visual approach for the concentration $i$-waves (left) together with the corresponding kinetic curves $\mathrm{F}_{i}(\mathrm{~T})$ (right) it is possible to consider the concentration $i$-waves propagation "in step" with the integral kinetic $\mathrm{F}_{i}(\mathrm{~T})$-curves behavior.

Concentration $i$-waves in the kinetic systems propagate without the formation of the concentration plateaus between the waves (see Figs. $2 \boldsymbol{a}, \boldsymbol{b}$, by way of example). In this case, due to a limited, and small size of the IEx matrix the concentration waves are unable to disperse with the formation of the concentration plateaus between the $i$-waves. Therefore, such kind of the stationary state with the plateaus for the waves is unattainable in the kinetic variants of the mass transport. However, in the case of the multi-component kinetics the important effect of the interference of the diffusion $i$-concentration waves of the $i$-components takes place $[1-4,7-9,12,13]$. Further, this behavior of the multi-component system with the $i$-waves interference are shown in the series of Figs. $2(\boldsymbol{a}, \boldsymbol{b})$ based on the approach of the multi-component kinetic mass balance equations (1) for the mass transport in the IEx matrix.

The description of the created generalized Model [1-4] has been followed by the computerized simulation of the multi-component $\mathrm{NC}$ system behavior. Here the partial case of the Model for the multicomponent IEx system is modeled. All the values and parameters $\left(D_{i}, \mathbf{r}, \mathbf{r o}, \mathrm{T}, \mathrm{X}_{i \mathrm{r}, \mathrm{ro},}\right)$ of the IEx system in the computer simulations are dimensionless (see Nomenclature). It is generalized the results of any modeling, including clear understanding of the process especially in Fundamentals. The obtained author's results of the computerized simulation for the multi-component mass transfer are original and new especially for the $i$-concentration waves behavior. The results of the modeling with the variants of the calculations for the $i$-waves interference are discussed and visually demonstrated, especially by the created author's animations.

\section{Concentration Waves in Multicomponent IEx Kinetics, Results of Their Interference}

The computerized simulation of the mass transfer in the multicomponent IEx kinetics by using the Model equations
(1) brings the calculation of the multi-component $i$-concentration distributions for the $i$-waves in the r-bead, and ro-fiber of the IEx matrixes together with the corresponding calculated kinetic $\mathrm{F}_{i}(\mathrm{~T})$ curves (Figs. 2a, b). The diffusion $i$-waves propagate along the dimensionless $\mathbf{r}$-, ro- distances of the IEx resin matrix in the course of the dimensionless time $\left(T=\mathrm{D}_{0} t / \mathrm{r}_{0}{ }^{2}\right)$ ). In course of Time $(\mathrm{T})$ the interference of the propagating $i$-concentration waves takes place during the multicomponent IEx kinetic process.

By the simulation there were calculated a number of the variants for the wide diapason of the various $D_{i}$-diffusivities of the ionic $i$-components. Among the all variants of the simulation the most interesting are the variants with the unusual non-monotonous kinetic curve $\mathrm{F}_{B}(\mathrm{~T})$ behavior (i.e. with the kinetic $\mathrm{F}_{B}$-peak). These variants are realized for the fast diffusion (with large $D_{B}$ ) of the $B$-component incoming into the r-bead, or ro-fiber from the outer solution. A number of such type of the kinetic $\mathrm{F}_{B}(\mathrm{~T})$-curves are considered in details in what follows (see Figs. 2a,b, right; and Figs. 3,4).

Figures 2 (a, $\boldsymbol{b}$, left sides) present the series of the pictures with the calculated propagating $i$-concentration waves along the $\mathbf{r}$-, ro- distances $\left(\mathrm{X}_{i \mathbf{r}, \text { ro }}\left(\mathrm{T}^{\mathrm{k}}\right)\right.$-profiles, $\boldsymbol{a}, \boldsymbol{b}$, left $)$ in course

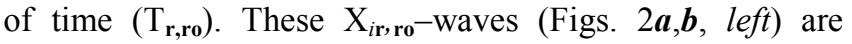
visually presented together, (i.e. in parallel -left, and right) with the integral kinetic $\mathrm{F}_{i r \text {,ro }}$-curves (Figs. 2a,b, right).

Here this original method of visual presentation of the computerized simulation of the IEx kinetics in course of time-T (Figures $2 \boldsymbol{a}, \mathbf{b}$, left and right) represents the effective interpretation of the results of the computerized modeling. The corresponding running moments $\mathrm{T}^{\mathrm{k}}\left(\mathrm{k}=1-4, \mathrm{~T}^{1}-\mathrm{T}^{4}\right)$ are marked by the white circles (o) placed in the kinetic $\mathrm{F}_{2 \mathbf{r}, \mathbf{r o}}(\mathrm{T})$ curves. (Figs. 2a, b, right, $\mathrm{T}_{\mathbf{r}}{ }^{1}-\mathrm{T}_{\mathbf{r}}{ }^{4}$, or $\mathrm{T}_{\mathbf{r o}}{ }^{1}-\mathrm{T}_{\mathrm{ro}}{ }^{4}$ )

In Figures $2 \boldsymbol{a}, \boldsymbol{b}$ the calculated $\mathrm{T}_{\mathbf{r}, \mathbf{r}}{ }^{1}-\mathrm{T}_{\mathbf{r}, \mathbf{r o}}{ }^{4}$ series for the running $\mathrm{T}^{\mathrm{k}}$-moments $(\mathrm{k}=1-4)$ show (but in $\mathrm{T}^{\mathrm{k}}$-fragments) the movement of the diffusion $i$-waves: $X_{i r, \mathbf{r o}}\left(T^{k}\right)$. The $i$-waves are presented "abreast" of the corresponding kinetic $\mathrm{F}_{i}\left(\mathrm{~T}^{\mathrm{k}}\right)$ - curves. In other words both pictures are presented together ("abreast") in Figs. $2 \boldsymbol{a}$ (for $\mathbf{r}$-bead): the left side for the $\mathrm{X}_{i r}\left(\mathrm{~T}_{\mathrm{r}}^{\mathrm{k}}\right)$-concentration waves; the right side - for the kinetic curve $\mathrm{F}_{\text {ir }}\left(\mathrm{T}_{\mathbf{r}}\right)$. In the same manner Figures $2 \boldsymbol{b}$ represent the $\mathrm{X}_{\text {iro }}\left(\mathrm{T}_{\mathrm{ro}}{ }^{\mathrm{k}}\right)$-curves (left) with the corresponding $\mathrm{F}_{\text {iro }}\left(\mathrm{T}_{\text {ro }}\right)$-curves (right) for the ro-fiber. Meanwhile the corresponding $\mathrm{T}_{\mathbf{r}, \mathbf{r o}}{ }^{1-4}$ moments are marked by white circles (o) in the corresponding kinetic $\mathrm{F}_{2 \mathrm{r}, \mathrm{ro}}\left(\mathrm{T}_{\mathrm{r}, \mathrm{r} \mathbf{0}}\right)$-curves (Figs. $2 \boldsymbol{a}, \boldsymbol{b}$, right). The "white" circle (0) in the kinetic $\mathrm{F}_{i}(\mathrm{~T})$-curve corresponds to the running k-moments $\mathrm{T}_{\mathbf{r}, \mathbf{r}}{ }^{1-4}$

So for the selected time moments $T_{r, r o}{ }^{1,4}$ the corresponding pair of k-points are marked by the "white" circles $(0)$ in the $F_{2 r, r o}(T)$-curves: $\left\{\mathrm{T}^{\mathrm{k}} ; \mathrm{F}_{2}{ }^{\mathrm{k}}\right\}$ in the Figures $2(\boldsymbol{a}, \boldsymbol{b} ;$ right $)$. This "circle" $(0)$ is placed in the $\mathrm{F}_{2 \mathrm{r}, \mathrm{r} \mathbf{r}}$-curves in correspondence with the evidence: running k-moments $\mathrm{T}^{1-4}$ on the $\mathrm{T}$ axes (see Figures $2 \boldsymbol{a}, \boldsymbol{b}$; right). The white "circle-point" $\left\{\mathrm{T}^{\mathrm{k}} ; \mathrm{F}_{2}{ }^{\mathrm{k}}\right\}$ corresponds to the relation: $\mathrm{F}_{2}{ }^{\mathrm{k}}=\mathrm{F}_{2}\left(\mathrm{~T}^{\mathrm{k}}\right)$. These "white" $\mathrm{k}$ - circles with the coordinates $\left\{\mathrm{T}^{\mathrm{k}} ; \mathrm{F}_{2}{ }^{\mathrm{k}}\right\}$ show the integral filling $\mathrm{F}_{2}{ }^{\mathrm{k}}$, having reached to the current situation $\mathrm{T}^{\mathrm{k}}\left(\mathrm{T}^{1}<\mathrm{T}^{2}<\mathrm{T}^{\mathrm{m}}<\mathrm{T}^{3}<\mathrm{T}^{4}\right)$ in accordance with 
the $\mathrm{X}_{i}\left(\mathrm{~T}^{\mathrm{k}}\right)$ - profiles.

Thus the whole kinetic process can be presented simultaneously and visually: by the $i$-waves distributions $\mathrm{X}_{i}\left(\mathbf{r}, \mathbf{r o} ; \mathrm{T}^{\mathrm{k}}\right)$ together with the corresponding position of the "circles-points": $\left\{\mathrm{T}^{\mathrm{k}} ; \mathrm{F}_{2}{ }^{\mathrm{k}}\right\}$ in the $\mathrm{F}_{2}(\mathrm{~T})$-curve, (Figs. 2a, $\boldsymbol{b}$, left, and right). Consider the locations of the "white" circles in any $\mathrm{F}_{2}(\mathrm{~T})$ - picture in Figures $2(\boldsymbol{a}, \boldsymbol{b}$, right $)$ it is seen that the two white circles (for $\mathrm{T}^{1}, \mathrm{~T}^{2}$ ) to the left from the kinetic maximum $\left(\mathrm{F}_{2}{ }^{\max }\right)$, and analogically the two circles (for $\left.\mathrm{T}^{3}, \mathrm{~T}^{4}\right)$ to the right from $\mathrm{F}_{2}{ }^{\max }$ show that the $\mathrm{T}_{\mathbf{r}, \mathbf{r}}{ }^{1-4}$-time values cover all the stages of the considered IEx kinetics: $\mathrm{T}^{1}<\mathrm{T}^{2}<\mathrm{T}^{\mathrm{m}}<\mathrm{T}^{3}<\mathrm{T}^{4}$.

The left series $\left(\mathrm{T}_{\mathbf{r}}^{1-4}\right)$ for the $\mathrm{X}_{i}\left(\mathbf{r} ; \mathrm{T}^{\mathrm{k}}\right)$-concentration waves in Figures $2 \boldsymbol{a}$ (r-distance), show the whole process (but in fragments) for the r-bead. Accordingly in the same manner the left series $\left(\mathrm{T}_{\mathbf{r o}}{ }^{1-4}\right)$ in Figures $2 \boldsymbol{b}$ (ro-distance) show it for the ro-fiber.

By the way, the calculated author's animations mentioned have obvious advantages in comparison with the fragmentary representation (in Figs. $2 \boldsymbol{a}, \boldsymbol{b}$ ), as the animations show the whole mass transfer process visually in course of time: $0<\mathrm{T}<\mathrm{T}^{\mathrm{fin}}$. In the same animation the $i$-waves propagate along the $\mathbf{r}$-, or ro-distances and interfere visually having on the right side ("abreast") the kinetic $\mathrm{F}_{2}(\mathrm{~T})$-curve with the "white circle-point" traveling along the $\mathrm{F}_{2}(\mathrm{~T})$-curve in correspondence with the running $\mathrm{T}$ moments, where at the first stage: $\mathrm{T}<\mathrm{T}^{\mathrm{m}}$, and then at the second stage $\mathrm{T}>\mathrm{T}^{\mathrm{m}}\left(\mathrm{T}^{\mathrm{m}}\right.$ is marked in the any animation by the color point, like in Figs. $2 \boldsymbol{a}, \boldsymbol{b}$, right).

The series of the joint $\left(\mathrm{X}_{i \mathbf{r}, \mathbf{r o}}(\mathrm{T})\right.$-left, and $\mathrm{F}_{i \mathbf{r}, \mathbf{r} \text { o }}(\mathrm{T})$-right $)$ pictures (Figs. 2a,b) represent the frames of the author's animations prepared for the visual interpretation of the computerized simulations in the oral computer presentations. The joint left, and right pictures with the $\mathrm{X}_{\text {ir, ro }}\left(\mathrm{T}^{\mathrm{k}}\right)$, and $\mathrm{F}_{\text {ir, ro }}\left(\mathrm{T}^{\mathrm{k}}\right.$ )-curves (Figs. 2a,b) are included into the animations as the frames for the corresponding current time situations: $T_{\mathbf{r}, \mathbf{r}} \mathrm{k}$. The animations in the full extent, and the fragmentary series $\left(\mathrm{T}^{1-4}\right)$ in Figures $2 \boldsymbol{a}, \boldsymbol{b}$ show visually the interference of the three $i$-waves, which occurs in the course of the ternary IEx diffusion kinetics in the spherical $\mathbf{r}$-bead (Figs. $2 \boldsymbol{a}$ ) or in the cylindrical ro-fiber (Figs. 2b).

The series $\left(\mathrm{T}_{\mathbf{r}, \mathbf{r} \boldsymbol{0}}{ }^{1-4}\right)$ in Figures $2 \boldsymbol{a}, \boldsymbol{b}$ show the details of the kinetic process for the ternary IEx system, where the $\mathrm{F}_{2(\mathrm{~B})}(\mathrm{T})$-curves (Figs. $2 \boldsymbol{a}, \boldsymbol{b}$, right) have the non-monotonous behavior in course of T-time. The series in Figures $2 \boldsymbol{a}\left(\mathrm{T}_{\mathbf{r}}{ }^{1-4}\right)$, and $2 \boldsymbol{b}\left(\mathrm{T}_{\mathrm{ro}}{ }^{1-4}\right)$ show the propagation in time $\left(\mathrm{T}^{1-4}\right)$, and the interference of the $i$-waves, which are dependent on the values of the $D_{i}$-diffusion coefficients. Besides, the $\mathrm{T}^{\mathrm{m}}$ (color points in Figs. $2 \boldsymbol{a}, \boldsymbol{b}$, right), and $\mathrm{T}^{\mathrm{fin}}$ values for the IEx kinetics are dependent additionally on the r,ro-shapes of the IEx matrix.

It is seen (Figs. $2 \boldsymbol{a}, \boldsymbol{b}$ ) that for the given diffusivities: $D_{B}=0.25>D_{A}=0.20>>D c=0.01$ there is appeared the considerable displacement effect with the $\mathrm{F}_{2 \mathbf{r}, \mathbf{r o}}{ }_{\max }$ -accumulation of the most mobile $B$-wave for the r-bead, and ro-fiber (Figs. 2a,b, right, the color circles in the

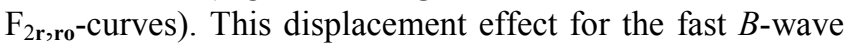

(Figs. 2a,b;left, $\mathrm{X}_{2 \mathrm{r}, \mathrm{ro}}$-dashed-dotted blue profiles) takes place under the certain conditions not only for the $\mathbf{r}$-bead but for the ro-fiber also (Figs. 2a,b, right, the color circles). It will be shown here the difference in the intensity of the displacement effect for the $\mathbf{r}$-bead in comparison with the ro-fiber.

In both $\mathbf{r}, \mathbf{r o - c a s e s}$ the displacement effect is located in the region of the smallest $C$-concentrations or in other words in frontal zone of the slow $C$-wave. This region of the $B$-accumulation in the $B$-wave is marked (Figs. $2 \boldsymbol{a}, \boldsymbol{b}$; left) by the vertical up arrows $(\boldsymbol{a})$ for the r-bead, and by the vertical dashed lines $(\boldsymbol{b})$ for the ro-fiber. The evidence for this location of the $B$-accumulation is the coincidence (along r-,ro- abscissa axes) between the position of the front (smallest $\left.X_{3 r, r o}-v a l u e\right)$ of the $X_{3 r, r o}$-profile with the corresponding marks for the $\mathrm{X}_{2 \mathrm{r}, \mathrm{ro}}\left(\mathrm{T}^{\mathrm{k}}\right)$-profiles: $(\boldsymbol{a}, \mathbf{r}$-, up arrows), and (b, ro-, dashed verticals). The comparison of the location of the marks for the $\mathrm{X}_{2 \mathrm{r}, \mathrm{ro}}$-waves and the location of the smallest $\mathrm{X}_{3 \mathrm{r}, \mathrm{ro}}$-values shows this fact distinctly (see Figs.2a,b; left, $\mathrm{X}_{2,3}$-waves). The reason for the $B$-accumulation in the fast $B$-wave (curve 2 ) is the displacement of this $B$-wave $\left(\mathrm{X}_{2 \mathbf{r}, \mathbf{r}}\right.$-curves, 2$)$ by the incursion of the front part of the another incoming (slow) $C$-wave $\left(\mathrm{X}_{3 \mathbf{r}, \mathbf{r}}-\right.$ curves, 3 ).

Figures $2 \boldsymbol{a}$ show that the especially significant $B$-accumulation occurs with the $i$-concentration jump (!) (left, up arrows) for the $\mathbf{r}$-bead $\left(\mathrm{F}_{2 \mathbf{r}}\right.$, right $)$, while the moderate $B$-accumulation occurs for the ro-fiber (Figs. $2 \boldsymbol{b}$, left, dashed verticals).

It means that the $B$-accumulation in the $\mathrm{X}_{2 r}$-wave for the r-bead is more intensive than for the ro-fiber. Meanwhile it is seen that the characteristic times $\left(\mathrm{T}_{\mathbf{r}, \mathbf{r} \mathbf{o}} \mathrm{m}, \mathrm{T}_{\mathbf{r}, \mathbf{r o}}{ }^{\text {fin }}\right)$ are smaller in the $\mathbf{r}$-bead in comparison with the ro-fiber $\left(\mathrm{T}_{\mathbf{r}}{ }^{\mathrm{m}}<\mathrm{T}_{\mathbf{r o}}{ }^{\mathrm{m}}, \mathrm{T}_{\mathbf{r}}^{\text {fin }}<\mathrm{T}_{\mathbf{r o}}{ }^{\text {fin }}\right.$, Figs. $2 \boldsymbol{a}, \boldsymbol{b}, \mathrm{F}_{2 \mathbf{r}, \mathbf{r},}$, right $)$. Thus due to the influence of these two oppositely directed factors ( $B$-accumulation intensity \& $\mathrm{T}_{\mathbf{r}, \mathbf{r o}}{ }^{\mathrm{m}}, \mathrm{T}_{\mathbf{r}, \mathbf{r o}}{ }^{\text {fin }}$ ) the total (integral) effect of the $B$-accumulation is approximately the same for the $\mathbf{r} \& \mathbf{r o}$ cases. Figures $2 \boldsymbol{a}, \boldsymbol{b}$ (right) show almost the same peaks with the $\mathrm{F}_{2 \mathbf{r}}{ }^{\max } \sim \mathrm{F}_{2 \mathbf{r o}}{ }^{\text {max }}$ values (the color points in Figs $2 \boldsymbol{a}, \boldsymbol{b})$ for the $\mathrm{F}_{2 \mathrm{r}, \mathbf{r}_{0}}$-curves.

Figures $2 \boldsymbol{a}, \boldsymbol{b}$ show that the $B$-accumulation in the $B$-wave occurs due to the $B$-displacement provided by the interference of the $B, C$-concentration waves. The $B$-accumulation in the fast $B$-wave is explained by the displacement effect due to the movement of the second slow invading $C$-wave $\left(D_{C}<D_{B}\right)$ with the incursion via the front part of the $\mathrm{X}_{3(\mathrm{C})}$-wave. The intensity of the displacement effect by the head of the slow $X_{3(C)}$-wave is more appreciable for the $\mathbf{r}$-bead than for the ro-fiber.

The physical sense of the $B$-concentration jump (Figs. $2 \boldsymbol{a}$, up arrows, left, series $\mathrm{T}_{\mathbf{r}}^{2-4}$ ) with the following $\mathrm{F}_{2 \mathrm{r}}{ }^{\text {max }}$-peak $(\boldsymbol{a}$, right $)$ for the $\mathbf{r}$-bead should be explained by the steep decrease $-d V_{r}$ of the $\mathbf{r}$-volume during the diffusion into the $\mathbf{r}$-sphere $\left(d V_{\mathbf{r}} \sim 4 \pi r^{2} d \mathbf{r}\right)$. The comparison with the $d V_{\text {ro }}$ volume for the diffusion into the ro-fiber, doesn't show such steep decrease $-d V_{\text {ro }}$ for the diffusion into the ro-fiber $\left(\mathrm{dV}_{\mathrm{ro}} \sim 2 \pi \mathrm{ro} * \mathrm{dro}\right)$. 
Therefore the $B$-accumulation for the ro-fiber is gradual and the $B$-concentration jump is absent visually (dashed lines in the series $\mathrm{T}_{\mathbf{r o}}{ }^{2}-\mathrm{T}_{\mathbf{r o}}{ }^{4}$ in Figs. 2b, left). So the displacement process for the $B$-wave is more intensive for the $\mathbf{r}$-bead but the $\mathrm{T}_{\mathbf{r}}{ }^{\mathrm{m}} \& \mathrm{~T}_{\mathbf{r}}$, ${ }^{\text {fin }}$ values are smaller than for the ro-fiber $\left(\mathrm{T}_{\mathbf{r o}}{ }^{\mathrm{m}} \& \mathrm{~T}_{\mathbf{r o}}{ }^{\text {fin }}\right.$, Figs. $2 \boldsymbol{a}, \boldsymbol{b}$, , left $)$. Thus in result $\mathrm{F}_{\mathrm{ir}, \mathbf{r o}}{ }^{\max }$ peak values in the integral $F_{i r, r o}(T)$-curves are displayed as almost the same (compare maxima: $F_{2 \mathbf{r}}{ }^{\max }(\boldsymbol{a}) \sim \mathrm{F}_{2 \mathbf{r o}}{ }^{\max }(\boldsymbol{b})$ in the kinetic $\mathrm{F}_{2 \mathbf{r}, \mathbf{r}}(\mathrm{T})$-curves, color circles, right).

(a) r-bead, $\quad \mathrm{T}_{\mathrm{r}}^{1}=0 ; \quad \mathrm{T}_{\mathrm{r}}^{2}=0.2 ; \mathrm{T}_{\mathrm{r}}^{3}=0.4 ; \quad \mathrm{T}_{\mathrm{r}}^{4}=0.8$
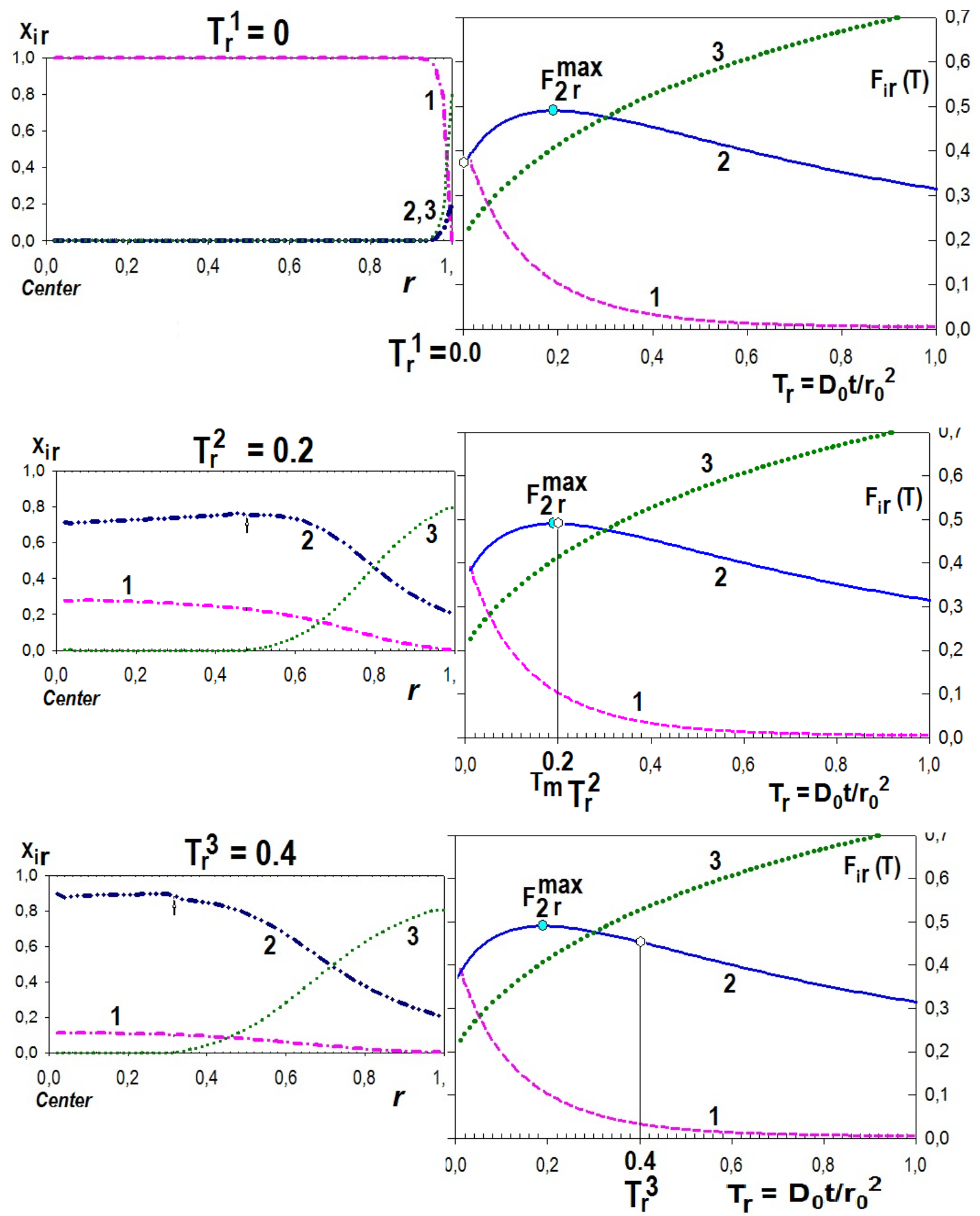


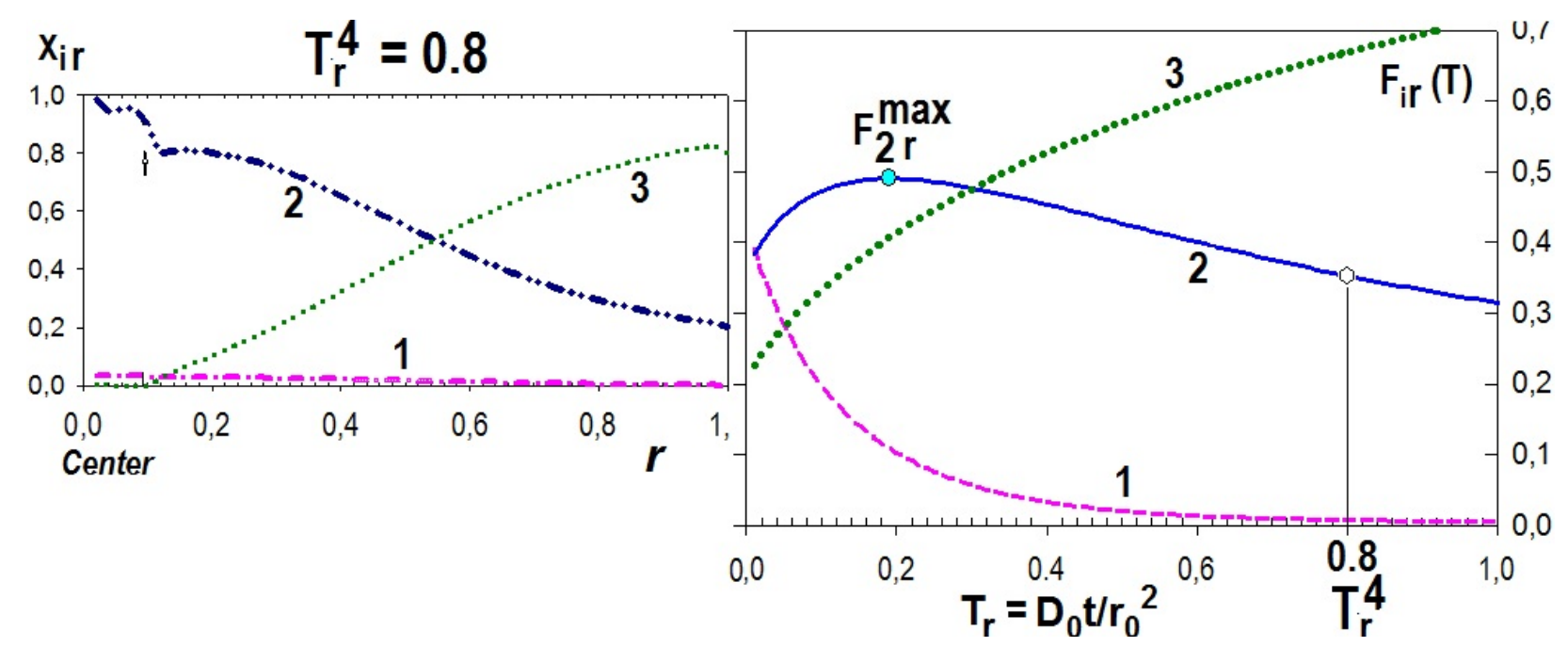

(b) ro-fiber; $\mathrm{T}_{\mathrm{ro}}^{1}=0 ; \quad \mathrm{T}_{\mathrm{ro}}{ }^{2}=[0.4] ; \quad \mathrm{T}_{\mathrm{ro}}{ }^{3}=[0.8] ; \quad \mathrm{T}_{\mathrm{ro}}{ }^{4}=[1.6]$
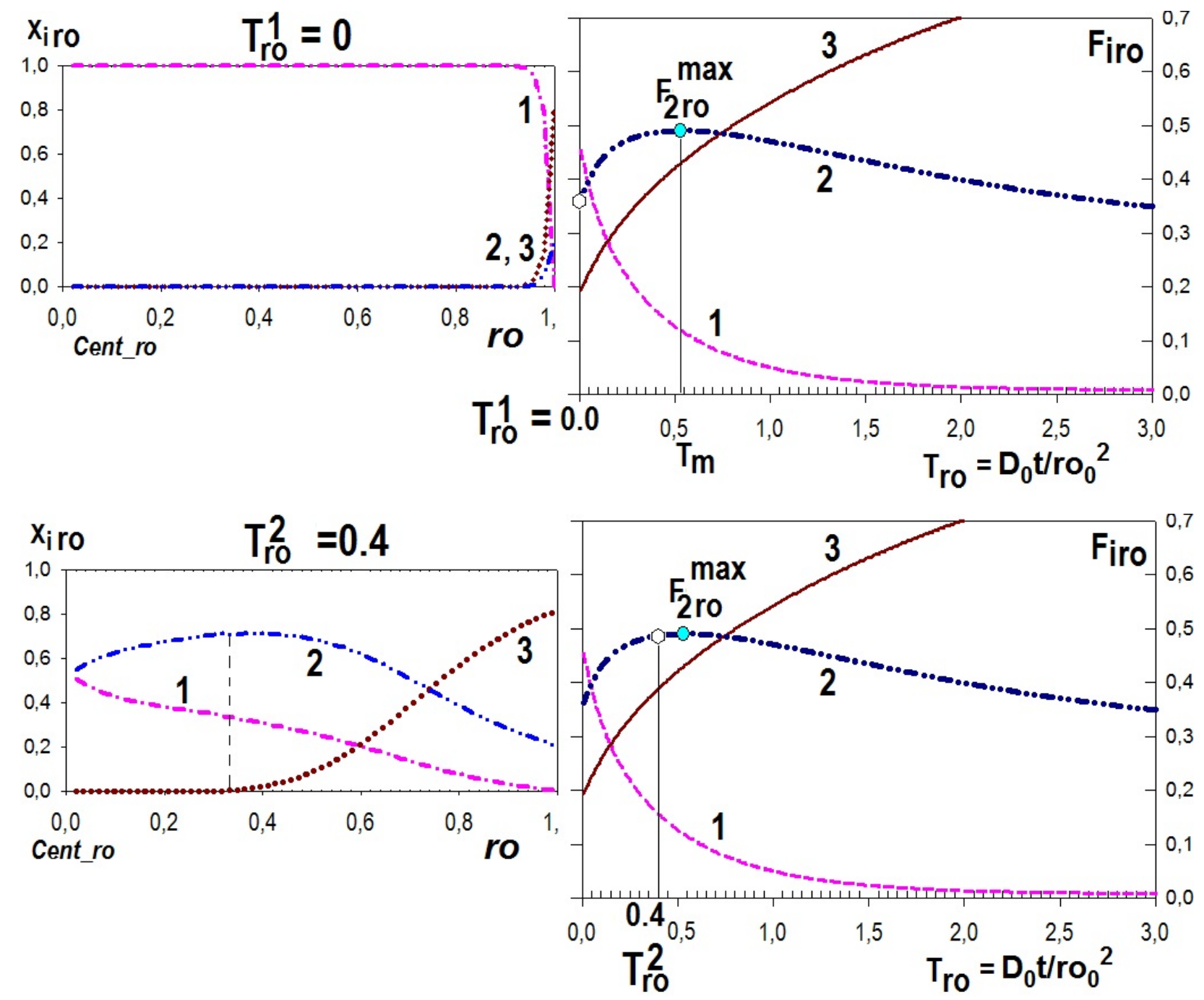

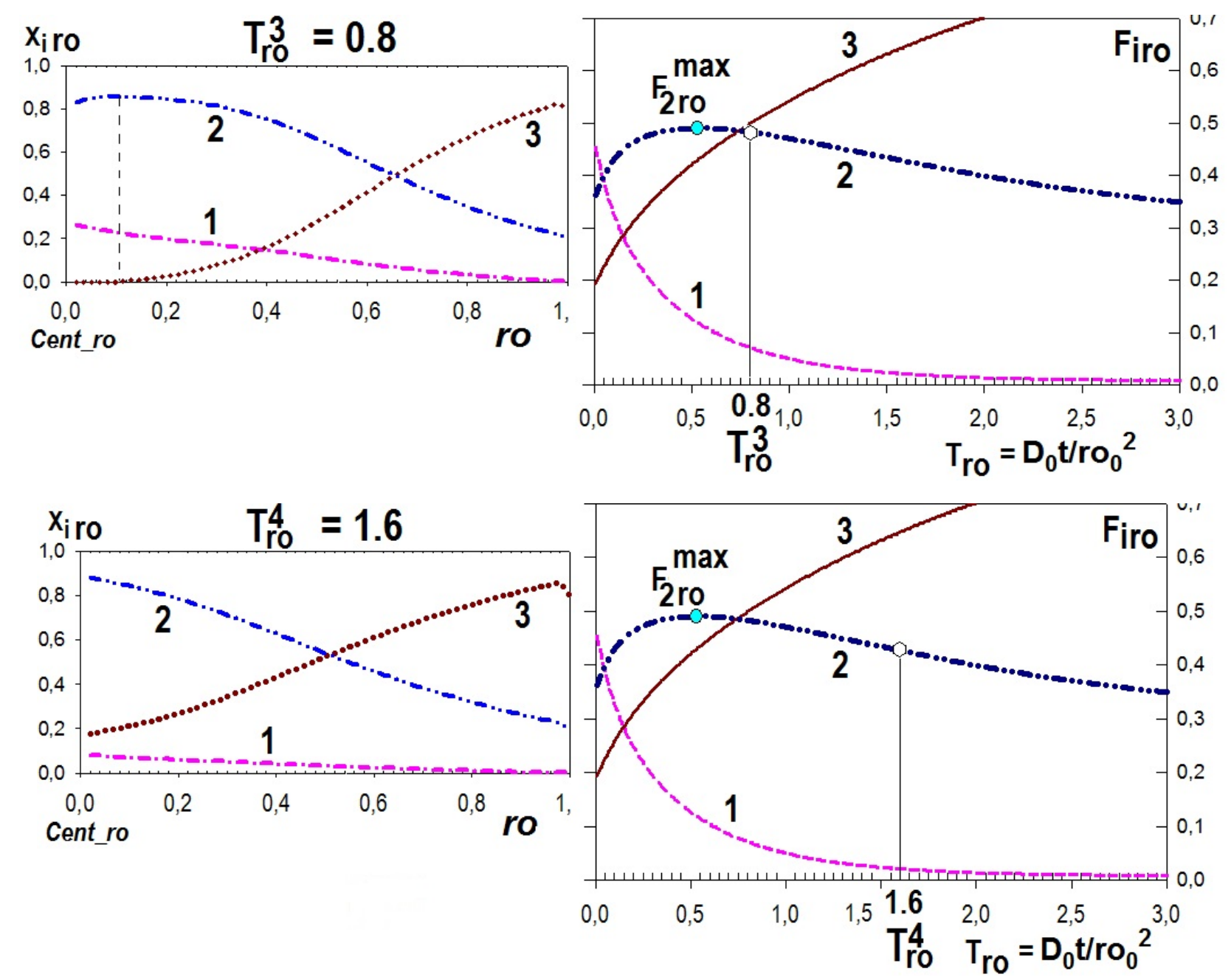

Figure 2(a,b). Propagation of $i$-waves along $\mathbf{r}(\boldsymbol{a})$, $\operatorname{ro}(\boldsymbol{b})$ - distances $(\boldsymbol{l e f t}):\left(\boldsymbol{a}\right.$, up: $\left.\mathrm{X}_{i}\left(\mathbf{r}, \mathrm{T}_{\mathbf{r}}{ }^{\mathrm{k}}\right), \mathrm{T}_{\mathbf{r}}{ }^{1}-\mathrm{T}_{\mathbf{r}}{ }^{4} ; 0<\mathrm{T}_{\mathbf{r}}<1.1\right) ;\left(\boldsymbol{b}, \operatorname{down}: \mathrm{X}_{\mathrm{i}}\left(\mathbf{r o}_{\mathbf{r}}, \mathrm{T}_{\mathbf{r o}}{ }^{\mathrm{k}}\right), \mathrm{T}_{\mathbf{r o}}{ }^{1}-\mathrm{T}_{\mathbf{r o}}{ }^{4} ; 0<\mathrm{T}_{\mathbf{r o}}<2.5\right)$. Kinetic curves $(r i g h t): \mathrm{F}_{i r}\left(\boldsymbol{a}\right.$, up); $\mathrm{F}_{\text {iro }}\left(\boldsymbol{b}\right.$, down). Times: $\mathrm{T}_{\mathbf{r}}{ }^{\mathrm{m}}=0.2(\boldsymbol{a}) ; \mathrm{T}_{\mathbf{r o}}{ }^{\mathrm{m}}=0.5(\boldsymbol{b}) ;(\boldsymbol{a}, \boldsymbol{b}) \mathrm{T}_{\mathbf{r},[\mathbf{r o} 0}{ }^{1-4}=0 ; 0.2,[0.4] ; 0.4,[0.8] ; 0.8,[1.6]$. Diffusivities: $D_{A}=0.20$, $D_{B}=0.25>>D c=0.01$. Big white $\mathrm{k}$-circles $\left\{\mathrm{T}^{\mathrm{k}}=\mathrm{T}^{1-4}, \mathrm{~F}_{2}{ }^{\mathrm{k}}\right\}$ in $\mathrm{F}_{2, \mathbf{r}, \mathbf{r} \mathbf{0}}$-curves. Start: $\mathrm{T}_{\mathbf{r}, \mathbf{r o}}{ }^{1}=0$; (Times $\mathrm{T}_{\mathbf{r}, \mathbf{r o}}{ }^{1-4}$, bold numerals on $\mathrm{T}_{\mathbf{r}, \mathbf{r o}}$ axes, $\mathrm{F}_{\mathrm{ir}, \mathbf{r} \mathbf{r},}$, right). Three $i$-ion-components, $i=1(A), 2(B), 3(C)$. Maximum accumulations $-\mathrm{F}_{2 \mathbf{r}, \mathbf{r o}}{ }^{\max }$ (color circles in $\mathrm{F}_{2 \mathbf{r}, \mathbf{r} 0}$-curves, right $, \boldsymbol{a}, \boldsymbol{b}$ )

The larger is the difference between diffusivities $\left(D_{B}-D_{C}\right)$ the more effective is the displacement for the $B$-wave. The calculations in the computerized simulations demonstrated that this condition for the $D_{B, C}$-diffusivities for the $B, C$-waves is not enough for the occurrence of the distinct kinetic peak. The $\mathrm{F}_{2(B)}{ }^{\text {max }}$-peak value depends essentially on the $D_{A}$-diffusivity for the $A$-wave outgoing from the IEx matrix: the larger is the $D_{A}$-diffusivity, the higher is the $\mathrm{F}_{2(B)}{ }^{\text {max }}$ peak (the color points) in the $\mathrm{F}_{2(B)}(\mathrm{T})$-curve (see for example Figs. $4 \boldsymbol{a}, \boldsymbol{b})$. The faster $A$-wave moves out of the IEx matrix, the smaller will be the influence of the $A$-wave on the total $i$-waves interference in the multicomponent IEx kinetics.

It means that the interference of all three diffusion $i$-waves plays the main role in the behavior of the kinetic $\mathrm{F}_{i}(\mathrm{~T})$-curves. Figures $2 \boldsymbol{a}, \boldsymbol{b}$ show that the shape of the resins (r-bead, or ro-fiber) also introduce the addition significant effect into the integral $\mathrm{F}_{2(B)}(\mathrm{T})$-curve behavior (physical sense see above). The r-.ro- shapes influence the times for the attainment of the final stage $\left(\mathrm{T}^{\mathrm{fin}}\right)$, including the time $\left(\mathrm{T}^{\mathrm{m}}\right)$ for the position of the kinetic maximum .
Figures $2 \boldsymbol{a}, \boldsymbol{b}($ right) show that the kinetic maximum $\mathrm{F}_{2(B)}{ }^{\max }$ (the color point) is reached much faster for the $\mathbf{r}$-bead (a,up) than for the ro-fiber $(\boldsymbol{b}$, down $)$. There are the corresponding relationships for the $\mathrm{T}^{\mathrm{m}}$ values: $\mathrm{T}_{\mathbf{r}}^{\mathrm{m}}(0,2)$ $<\mathrm{T}_{\mathbf{r o}}{ }^{\mathrm{m}}(0.5)$ for the $\mathrm{F}_{2 \mathbf{r}, \mathbf{r o}}{ }^{\max }$ points with the corresponding relation: $\mathrm{F}_{2 \mathbf{r}}{ }^{\max } \sim \mathrm{F}_{2 \mathbf{r o}}{ }^{\max }$ (Figs. 2a, b, right).

The comparison of the kinetic curves $\mathrm{F}_{i \boldsymbol{r}, \text { ro }}(\mathrm{T})$ (Figs. 2a, $\boldsymbol{b}$, right) shows that the duration $\left(\mathrm{T}^{\mathrm{fin}}\right)$ of the whole diffusion kinetic process is much shorter for the $\mathbf{r}$-bead than for the ro-fiber: $\quad\left(\mathrm{T}_{\mathbf{r}} \mathrm{fin}_{1} \sim 1.\right),(\boldsymbol{a})<\left(\mathrm{T}_{\mathbf{r o}}{ }_{\mathrm{fin}} \sim 2.\right),(\boldsymbol{b}) . \quad$ These relations support the fact that that the displacement effect is more intensive for the $\mathbf{r}$-bead than for the ro-fiber, (see

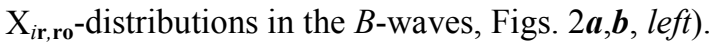

The $B$-accumulation in the $\mathrm{X}_{2(B)}$-wave for the IEx r,ro-resins is demonstrated visually via the author's computerized animations mentioned. The necessary conditions for the intensive displacement effect is determined by the inequality relation for the diffusivities: $\left(D_{B}, D_{A}\right)>>D_{C}$.

Thus under the certain conditions (i.e., for the big difference in the diffusivities $\left.D_{B, A}>>D_{C}\right)$ the $B$-component 
is accumulated exactly in the region of the front part for the smallest concentrations of the slow $\mathrm{C}$ - wave (see the dashed blue $\mathrm{X}_{i \mathrm{r}}, \mathrm{X}_{\text {iro }}$-profiles for the $B$-waves, Figs. $2 \boldsymbol{a}, \boldsymbol{b}$, left). The position $\left(\mathrm{T}^{\mathrm{m}}\right)$ for the $\mathrm{F}_{\text {ir,ro }}{ }^{\max }$-point (the color circle) and its value are dependent on the relationships for the values of the $D_{B} \sim D_{A} \sim D_{C}$ diffusivities (Figs. 2a, b, right).

\subsection{Multicomponent IEx Kinetic Curves, Results of $i$-Concentration Waves Interference}

A number of the ternary IEx systems describing the multicomponent kinetic behavior in the mono-functional IEx matrix of the various shapes (r-bead, ro-fiber) were included into the consideration. The computerized simulations of the IEx systems for a number of variants with the wide diapason of the $\mathrm{D}_{i}$ diffusivities were realized by a computer on the basis of the author's computer programs. In result of the computer simulation there were calculated the $\mathrm{X}_{i}(\mathbf{r}, \mathbf{r o}$; $\mathrm{T}$ )-waves (profiles) moving along the $\mathbf{r}$-,or ro- distances in course of time $(\mathrm{T})$. Besides the corresponding integral kinetic $\mathrm{F}_{\text {ir, ro }}(\mathrm{T})$-curves have been calculated simultaneously during the simulation. The examples of the simulation for the two cases of the calculation of the $\mathbf{X}_{\mathrm{ir}, \mathrm{ro}}(\mathrm{T})$-waves, including in parallel $F_{i r},{ }_{0}(T)$-curves in the full "computerized experiment" are presented visually in Figures $2(\boldsymbol{a}, \boldsymbol{b})$. A number of the kinetic $F_{i r, r o}(T)$-curves for the wide diapason of the $\mathrm{D}_{i}$-diffusivities are presented below (Figs. 3,4). It was fulfilled the investigation of the influence of the $\mathrm{D}_{i}^{l}$-diffusivities values on the $\mathrm{F}_{l}$-kinetic curves with the non-monotonous behavior (examples in Figs. $2 \boldsymbol{a}, \boldsymbol{b}$, right, and Figs. 3, and 4).

\subsubsection{Kinetic Curves with the Non-monotonous Time Behavior for the nonselective IEx}

Ternary diffusion kinetics with the $\mathrm{X}_{\mathrm{i}, \mathrm{r}, \mathrm{r} \text {-concentration }}$ waves for the IEx system: RA/(B+C) inside the nonselective IEx matrix are considered above. In this particular but important case there is no reactions and thus the IEx matrix is usual and mono-functional. So in this case it is considered rather simple ternary IEx kinetics in the IEx matrix with the three $i$-concentration waves propagating in course of time (Figs. 2a, $\boldsymbol{b}, \mathrm{X}_{i \mathbf{r}, \mathbf{r o}}$-profiles, left). The boundary and initial conditions correspond to the ternary IEx: $\mathrm{RA}_{\text {resin }} /(\mathrm{B}+\mathrm{C})_{\text {solution, }}$, where $B, C$-ions are invading from the outer solution into the IEx resin, which is initially in the form of the $\mathrm{A}^{+}$-ions (RA).
In the result of the ternary IEx: $\mathrm{RA} /(\mathrm{B}+\mathrm{C})$ there are formed two incoming $\mathrm{B}, \mathrm{C}$-waves and one outgoing A-wave (Figs. $\left.2 \boldsymbol{a}, \boldsymbol{b}, \mathrm{X}_{i}, l e f t\right)$. The interference of the three $i$-concentration $\mathrm{X}_{i}$-waves (Figs. $2 \boldsymbol{a}, \boldsymbol{b}$, left) occurs in the course of ternary diffusion mass transfer inside the IEx matrix. Figures $2 \boldsymbol{a}, \boldsymbol{b}$, $\left(\mathrm{F}_{i}(\mathrm{~T})\right.$, right $)$ show the corresponding three kinetic $\mathrm{F}_{i}(\mathrm{~T})$ -curves in the r-bead, and ro-fiber: $\mathrm{F}_{\text {ir }}(\mathrm{T}),(\boldsymbol{a})$ and $\mathrm{F}_{\text {iro }}(\mathrm{T}),(\boldsymbol{b})$.

Moreover the results of the computerized simulation for the wide diapason of $D_{i}$-diffusivities are obtained. Here the dependences of the kinetic $\mathrm{F}_{i}(\mathrm{~T})$-curves behavior will be discussed in dependence of the various diffusivity $D_{i}^{l}$ -values $\left(D_{i}^{I}, D_{i}^{2}, D_{i}^{3}\right)$.

Figures $3(\boldsymbol{a}, \boldsymbol{b}),\left(D_{B}^{l}\right.$, Variants 1r,1ro-3r,3ro) show the $\mathrm{F}_{\mathrm{ir}, \mathrm{ro}}(\mathrm{T})$-curves behavior in dependence of the various values of the $D_{B}^{l}$-diffusivity.

Figures $4(\boldsymbol{a}, \boldsymbol{b}),\left(D_{A}^{l}\right.$, Variants 1r,1ro-3r,3ro) show the analogical dependences of the $\mathrm{F}_{\text {ir, ro }}(\mathrm{T})$ - curves behavior for the various values of the $D_{A}^{i}$-diffusivity.

For the $F_{B}(\mathrm{~T})$ kinetic curves there is the reduction of the $\mathrm{F}_{2(B)}{ }^{\text {max }}$-value with the decrease of the $D_{B}$-diffusivity. The same type dependence occurs for the decrease of the $D_{A^{-}}$ diffusivity.

The decrease of the $\mathrm{F}_{2 \mathbf{r}, \mathbf{r}}{ }^{\text {max }}$-values (the color circles) with the $D_{B}^{l}$ (Figs. 3) or $D_{A}^{l}$ (Figs. 4) diffusivity reduction from the large- $D_{B}{ }_{B}$, till the small- $D_{B}^{3}$ (Figs. $3 \boldsymbol{a}, \boldsymbol{b}, D_{B}$, variants 1r,1ro-3r,3ro), or from the large $D_{A}^{I}$, till the small $D_{A}^{3}$ (Figs. $4 \boldsymbol{a}, \boldsymbol{b}, D_{A}$, variants $\left.1 \mathrm{r}, 1 \mathrm{ro}-3 \mathrm{r}, 3 \mathrm{ro}\right)$ means the corresponding reduction of the $B$-displacement effect. Consequently the obtained monotonic dependences ${F_{B}}_{B}{ }^{\text {ax }} \sim \mathrm{D}_{B, A}$ (compare Figs. 3,4, left and right) show that the further $\mathrm{D}_{B, A}$ reduction till the relations $D_{B}(<0.05)$, or $D_{A}(<0.05)$ brings the transition from the non-monotonous kinetic $\mathrm{F}_{2 \mathbf{r}, \mathbf{r o}}(\mathrm{T})$ - curve behavior (Figs. $3,4$, left, variants $1 \mathrm{r}, 1 \mathrm{ro})$ to the almost monotonic $\mathrm{F}_{2 \mathrm{r} \text {,ro }}$ (T)-curve behavior (Figs. 3,4, right, variants 3r,3ro).

Thus it is seen that the decrease of the $D_{B}$ (or $D_{A}$ )-diffusivity reduces the displacement effect for the $B$-concentration wave. When $D_{B}$ (or $\left.D_{A}\right)$-value becomes smaller than 0.05 the non-monotonous $\mathrm{F}_{2}(\mathrm{~T})$-curve behavior is impossible even for the big $\mathrm{D}_{\mathrm{A}}\left(\right.$ or $\left.D_{B}\right)$ value (Figs. 3, or 4, right). In this cases the $B, C$-waves move in the IEx matrix along the r,ro-distances not far from each other and the three $B, C \& \mathrm{~A}$-concentration waves interfere together with the minimum of the displacement effect for the $B$-wave $\left(\mathrm{X}_{2 \mathbf{r}, \mathbf{r}}\right)$. 
(a) r-bead, $\mathrm{F}_{\mathrm{ir}}(\mathrm{T}), \quad D_{B}{ }^{1}=0,25(1 \mathrm{r}) ; \quad D_{B}{ }^{2}=0.15(2 \mathrm{r}) ; \quad D_{B}{ }^{3}=0.05(3 \mathrm{r})$

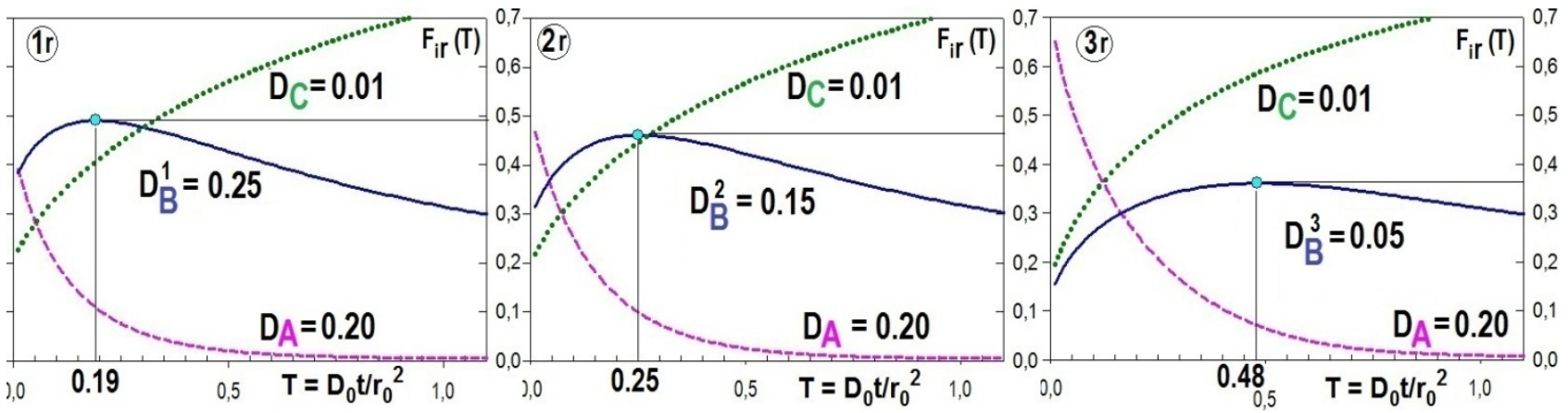

(b) ro-fiber, $\mathrm{F}_{\text {iro }}(\mathrm{T}), D_{B}{ }^{1}=0,25(1 \mathrm{ro}) ; \quad D_{B}{ }^{2}=0.15(2 \mathrm{ro}) ; \quad D_{B}{ }^{3}=0.05(3 \mathrm{ro})$

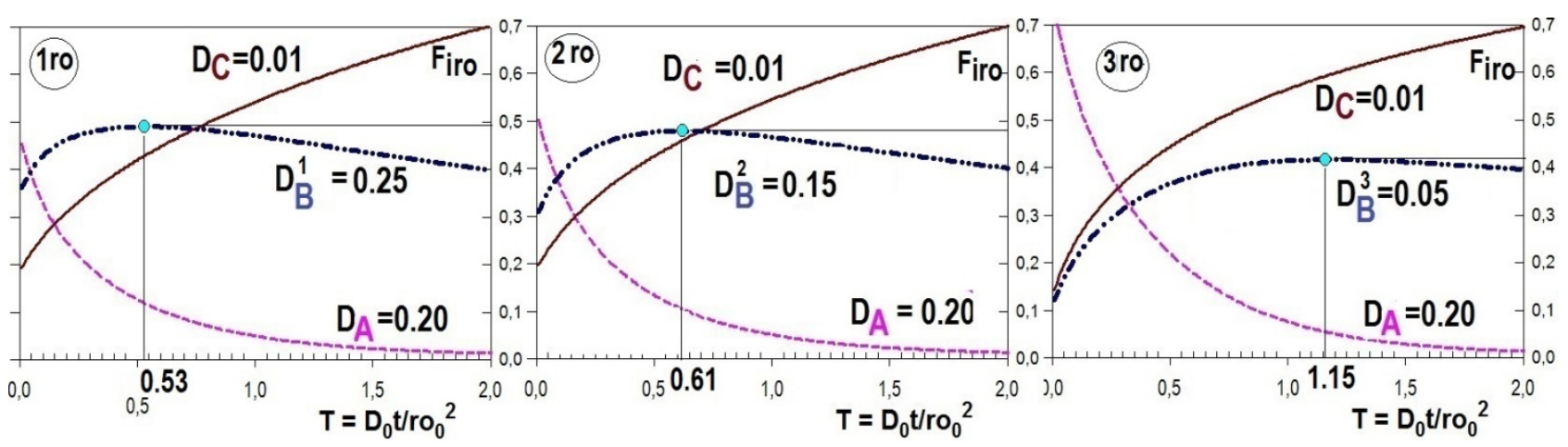

Figure $3(\boldsymbol{a}, \boldsymbol{b})$. Behavior of the kinetic curves $\mathrm{F}_{i \mathbf{r}, \mathbf{r}}(\mathrm{T})$ in dependence of $\mathrm{D}_{\mathrm{B}}$ value: $\mathbf{r}$-bead ( $\boldsymbol{a}$, Variants: $\left.1 \mathrm{r}-3 \mathrm{r}\right)$; ro-fiber $\left(\boldsymbol{b}\right.$, Variants: 1ro-3ro). $\mathrm{T}_{2 \mathbf{r}}{ }^{\mathrm{mm}}$ $=0.19(1 \mathrm{r}), 0.25(2 \mathrm{r}), 0.48(3 \mathrm{r}) ; \mathrm{T}_{2 \mathbf{r o}}{ }^{\mathrm{mm}}=0.53(1 \mathrm{ro}), 0.61(2 \mathrm{ro}), 1.15(3 \mathrm{ro})$. Various $D_{B}$-diffusivities: $D_{B}{ }^{1}=0.25, D_{B}{ }^{2}=0.15, D_{B}{ }^{3}=0.05 ; \quad D_{A}=0.20 ; \quad D_{C}=0.01$. The points $\left\{\mathrm{T}^{\mathrm{m}} ; \mathrm{F}_{2}{ }^{\max }\right\}$ are marked by color circles on the kinetic $\mathrm{F}_{2 \mathrm{r}, \mathrm{r} \text { - }}$-curves.

(a) $\quad$-bead, $\quad \mathrm{F}_{\text {ir }}(\mathrm{T}), \quad D_{A}{ }^{1}=0.15(1 \mathrm{r}) ; \quad D_{A}{ }^{2}=0.10(2 \mathrm{r}) ; \quad D_{A}{ }^{3}=0.05(3 \mathrm{r})$

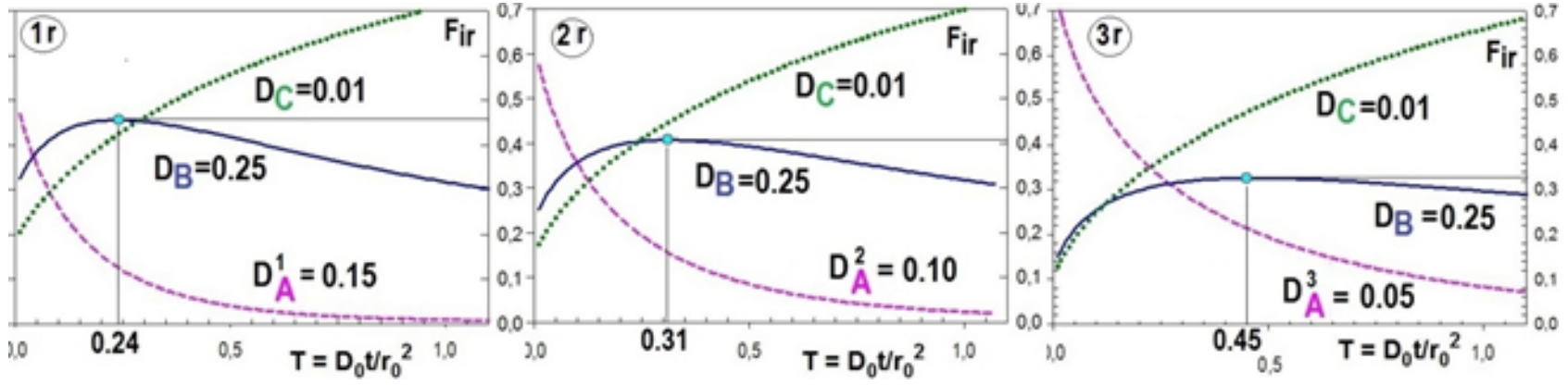

(b) ro-fiber, $\mathrm{F}_{\text {iro }}(\mathrm{T}), \quad D_{A}{ }^{1}=0.15(1 \mathrm{ro}) ; \quad D_{A}{ }^{2}=0.10(2 \mathrm{ro}) ; \quad D_{A}{ }^{3}=0.05$ (3ro)

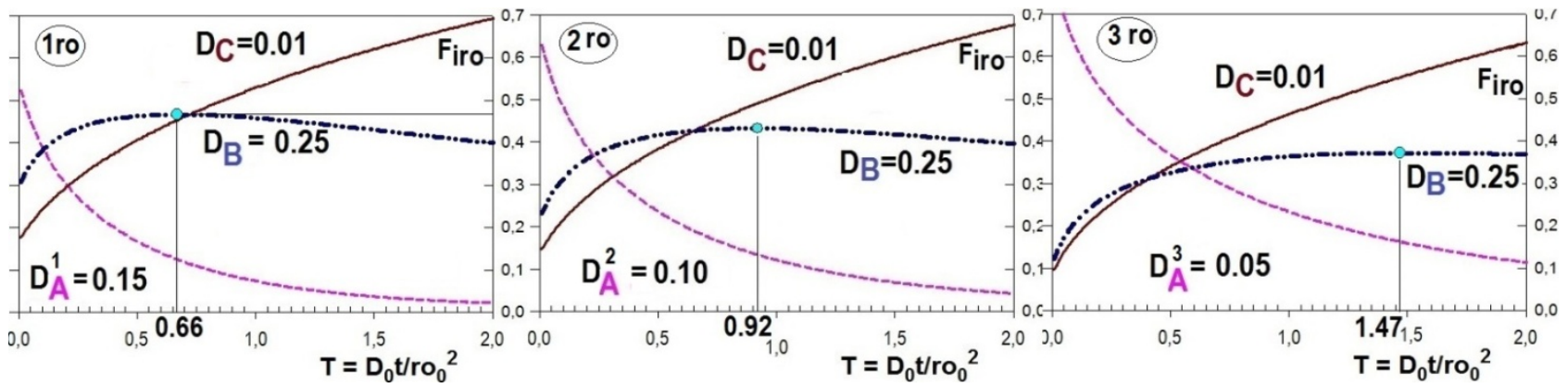

Figure $4(\boldsymbol{a}, \boldsymbol{b})$. Behavior of the kinetic curves $\mathrm{F}_{i \mathbf{r}, \mathbf{r o}}(\mathrm{T})$ in dependence of $\mathrm{D}_{\mathrm{A}}$-value: $\mathbf{r}$-bead $\quad(\boldsymbol{a}$, Variants: $1 \mathrm{r}-3 \mathrm{r})$; ro-fiber $(\boldsymbol{b}, \mathrm{Variants}$ : 1 ro-3ro $)$. $\mathrm{T}_{2 \mathbf{r}} \mathrm{mm}_{=}=0.24$ (1r); $0.31(2 \mathrm{r}) ; 0.45(3 \mathrm{r}) ; \mathrm{T}_{2 \mathrm{ro}}{ }^{\mathrm{mm}}=0.66$ (1ro); 0.92 (2ro); 1,47(3ro). Various $D_{A}$ diffusivities: $D_{A}{ }^{1}=0.15, D_{A}{ }^{2}=0.10, D_{A}{ }^{3}=0.05 ; D_{B}=0.25 ; \quad D_{C}=0.01$. The points $\left\{\mathrm{T}^{\mathrm{m}} ; \mathrm{F}_{2}{ }^{\max }\right\}$ are marked by color circles on the kinetic $\mathrm{F}_{2 \mathbf{r}, \mathbf{r o}}$-curves. 


\section{Conclusions}

The modern kinetic Model for the multicomponent mass transfer in the IEx non-selective materials is used for the investigation of the $i$-component concentration waves behavior in the partial case of the Diffusion Model: multicomponent mass transfer for the nonselective IEx kinetics: $\mathrm{RA}_{\text {resin }} /\left(\mathrm{B}^{+}+\mathrm{C}^{+}\right)_{\text {solution }}$. The computerized simulations of the multicomponent nonlinear IEx systems is fulfilled on the basis of the mass balance equations of the Model including influence of the electric field inside the IEx matrix. The coupled influence of the $i$-concentration gradient together with the electric field force is included into the consideration due to the well known classical Nernst-Plank relations for the $\mathrm{J}_{i}$-fluxes.

The simulation brings the computerized calculation of the propagating multicomponent $i$-concentration waves. Additionally the integral kinetic $\mathrm{F}_{i}(\mathrm{~T})$-curves in the $\mathbf{r}$-bead or ro-fiber are calculated in parallel during the simulation of the nonselective IEx kinetics. Thus the new results are obtained, which describe the behavior of the multicomponent $\mathrm{X}_{i}(\mathbf{r}, \mathbf{r o}$; $\mathrm{T})$ concentration waves together with the kinetic $\mathrm{F}_{\text {ir,ro }}(\mathrm{T})$-curves. The propagation of the $i$-waves, and the kinetic $\mathrm{F}_{i}(\mathrm{~T})$-curves behavior is determined by the various $D_{i}$ -diffusivities values $(i=A, B, C)$ of the $i$-components diffusing in the two various shapes of the IEx resins: the r-bead, and ro-fiber.

1. Due to the $i$-waves interference the noval displacement effect (activated by the slow $C$-wave) takes place for the propagation of the fast diffusion $B$-wave: $\mathrm{X}_{B}(\mathbf{r}, \mathbf{r o} ; \mathrm{T})$ for the incoming $B$-ions with the large diffusivity $D_{\mathrm{B}}\left(D_{\mathrm{B}}>>D_{\mathrm{C}}\right)$ in the resin matrix for the both shapes. This new displacement effect for the $B$-wave is the origin for the anomalous, non-monotonic behavior of the kinetic curves $-\mathrm{F}_{B}(\mathrm{~T})$ with the availability of the kinetic $\mathrm{F}_{2 \mathbf{r} \text {,ro }}{ }^{\max }$-maximum. Both characteristics of the such type kinetic curves: $F_{B r \text {,ro }}{ }_{\max }$ value and its position $\left(\mathrm{T}^{\mathrm{m}}\right)$ on the $\mathrm{T}$-abscissa axes depend on the relations for the $D_{i}$-diffusivities: $D_{A} \sim D_{B} \sim D_{C}$ :

- The larger are the $D_{i}$-diffusivities for the incoming and outgoing counterions $B^{+}, A^{+}\left(D_{B}, D_{A}\right)$ the larger is the kinetic maximum $-\mathrm{F}_{B}$; ${ }^{\max }$

- The larger are $D_{B}, D_{A}$ - diffusivities, the more significant is the displacement effect influence (especially when $\left.D_{B}>>D_{C}\right)$;

- The small difference in the $D_{B}, D_{C}$-diffusivities accounts for the trend to the usual kinetic $\mathrm{F}_{\mathrm{B}}(\mathrm{T})$ - curve monotonic behavior.

2. The displacement of the fast incoming $B$-wave is realized due to the incursion of the second, (and slow) $C$-wave $\left(D_{B}>>D_{C}\right)$. The position of the $B$-accumulation in the $B$-wave coincide with the head of the slow $C$-wave. Therefore the location of the displacement effect is positioned at the region of the small (around zero) concentrations of the $C$-wave. The cause and $C$-front location for the displacement is seen especially clearly for the r-bead, where the forming concentration jump in the $B$-wave is positioned at the head of the $C$-wave (Figs. $2 \boldsymbol{a}$, $\mathrm{X}_{2 \mathrm{r}}$, left).

3. The displacement of the $B$-wave by the second incoming slow $\mathrm{C}$-wave brings the accumulation of the $B$-concentration in the r-bead or ro-fiber. This $B$-accumulation becomes apparent as the $\mathrm{F}_{B}{ }^{\max }$ maximum: (the color circles) in the kinetic $\mathrm{F}_{\text {ir,ro-curves (Figs. }}$ $2 \boldsymbol{a}, \boldsymbol{b}$, right $)$. The kinetic $\mathrm{F}_{B}{ }^{\text {max }}$-maximum and its position $\left(\mathrm{T}^{\mathrm{m}}\right)$ on the $\mathrm{T}$-axes are dependent on the relations for the $D_{i}$-diffusivities.

4. The availability of the displacement effect for the $B-$ wave $\left(\mathrm{X}_{2 \mathrm{r}, \mathrm{ro}}\right)$ is demonstrated visually by the author's computerized animations for the $\mathrm{X}_{B}(\mathbf{r}, \mathbf{r o} ; \mathrm{T})$-waves in the r-bead, or ro-fiber. The computerized animations are constructed by the frames obtained as the results of the computer simulation on the basis of the mass balance partial differential equations for the $i$ - waves (by application of the key $\mathrm{W}^{+}$concept).

5. The "multicomponent concentration wave" concept $\left(\mathrm{W}^{+}\right)$shows its effectiveness in the study of the multicomponent mass transfer in the IEx matrix with the wave broadening Diffusion factor $\left(\mathbf{I I}, D_{\mathrm{A}}, D_{\mathrm{B}}, D_{\mathrm{C}}\right)$. It brings the clear, and understandable treatment of the multicomponent mass transfer kinetics inside the IEx matrix. The obtained original, new results show the behavior of the broadening and interfering propagating $i$-concentration waves during the multicomponent mass transfer IEx kinetics.

\section{Nomenclature}

\begin{tabular}{|c|c|c|}
\hline Symbol & Meaning & Units \\
\hline $\mathrm{T}=$ & $\mathrm{D} 0 * \mathrm{t} / \mathrm{r} 02, \mathrm{D} 0 * \mathrm{t} /(\mathrm{ro} 02)-\operatorname{time}(\mathrm{t})$ & dimensionless \\
\hline $\mathrm{Di}$ & Diffusion coefficients (relative to scale - D0) & dimensionless \\
\hline $\mathrm{r}=$ & $\mathrm{r} / \mathrm{r} 0$ - distance $\mathrm{r}$ along radius $\mathrm{r} 0$ of the spherical $\mathrm{r}$-bead, $0<\mathrm{r}<1$ & $\mathrm{r}$, dimensionless \\
\hline $\mathrm{ro}=$ & ro/ro0 - distance ro along radius ro 0 of the cylindrical ro-fiber, $0<$ ro $<1$ & ro, dimensionless \\
\hline HETP & $\begin{array}{l}\text { Height of Equivalent Theoretical Plate (HETP, }[\mathrm{cm}]) \text { - parameter for the broadening } \\
\text { concentration waves in Theory of Chromatography }\end{array}$ & $\mathrm{cm}$ \\
\hline
\end{tabular}




\begin{tabular}{|c|c|c|}
\hline $\mathrm{Xi}, \mathrm{j}$ & concentrations of i,j-components (relative to input conc.- $\mathrm{C} 0$ ) & dimensionless \\
\hline $\mathrm{NC}$ & NanoComposites & \\
\hline $\mathrm{i}$ & index, number of $\mathrm{i}$ - component , $\mathrm{i}=1(\mathrm{~A}), 2(\mathrm{~B}), 3(\mathrm{C})$ & \\
\hline Fir,ro & $\begin{array}{l}\text { Fir(T), Firo (T) - integral kinetic i-curves for the shapes: r-bead, or ro-fiber (Figs. 2a,b, } \\
\text { right, and Figs.3,4) }\end{array}$ & dimensionless \\
\hline F2r,rok & $\begin{array}{c}\text { F2k }=\text { F2(Tk) - selected k-points }\{\text { Tk; F2k }\} \text { in the kinetic F2(T)-curve marked by the "big } \\
\text { white" circles in the pictures (series of Figs. 2a,b, right) }\end{array}$ & dimensionless \\
\hline Tr,rok & $\begin{array}{l}\text { Trk, Trok }(\mathrm{k}=1,2,3,4)-- \text { selected current situations -Times }(\mathrm{T} \text { r,ro1-4), for the attainment } \\
\text { of the k-point in the kinetic F2r,rok - curves (Figs. 2a,b, right) for the shapes: r-bead, and } \\
\text { ro-fiber; F2k = F2(Tk) }\end{array}$ & dimensionless \\
\hline F2r,romax & $\begin{array}{l}\text { F2rmax }=F 2 \text { r }(\mathrm{T} \mathrm{rm}), \mathrm{F} 2 \text { romax }=\mathrm{F} 2 \text { ro }(\text { Tro } \mathrm{m}) \text { - maximum values in the kinetic Fi(T)-curves } \\
(\text { The points }\{\mathrm{Tm} ; \mathrm{F} 2 \mathrm{max}\} \text { marked by color points, Figs. } 2-4) \text { for various IEx shapes: r-bead, } \\
\text { and ro-fiber }\end{array}$ & dimensionless \\
\hline Tr,rom & $\begin{array}{c}\text { Trm, Trom } \begin{array}{c}(\text { Trm<Trom }) \text { - Time for the attainment of the kinetic maximum- F2r,romax } \\
\text { for the IEx shapes: r-bead, and ro-fiber }\end{array} \\
\end{array}$ & dimensionless \\
\hline $\mathrm{Xj}$ & Concentrations of $j$-component ; IEx systems ; computerized IEx kinetics simulation & dimensionless \\
\hline Xir, Xiro, Xir,ro & $\begin{array}{c}\mathrm{Xi}(\mathrm{r} ; \mathrm{T}) \mathrm{Xi}(\mathrm{ro} ; \mathrm{T}) \mathrm{Xi}(\mathrm{r}, \mathrm{ro} ; \mathrm{T}) \text { - concentration profiles of the } \mathrm{i} \text { - component waves in the } \\
\text { r-bead, or ro-fiber; } \mathrm{i}=\mathrm{A}(1), \mathrm{B}(2), \mathrm{C}(3)\end{array}$ & dimensionless \\
\hline NP & NanoParticles (NP) inside NanoComposite matrix & \\
\hline $\mathrm{F}, \mathrm{R}, \mathrm{T}$ & $\begin{array}{l}\text { Faraday constant, R-gas constant, T-Kelvin temperature in classical Nernst-Plank (N-P) } \\
\text { relationship for the Ji -fluxes }\end{array}$ & dimensionless \\
\hline $\mathrm{n}$ & $\mathrm{n}$-components in IEx systems for modeling & \\
\hline $\mathrm{Ji}$ & Fluxes for the mass of the i-components & \\
\hline $\mathrm{n}=3$ & $\begin{array}{l}\text { Ternary IEx kinetic system, ionic components, } \\
\qquad \mathrm{i}=1(\mathrm{~A}), 2(\mathrm{~B}), 3(\mathrm{C})\end{array}$ & \\
\hline SUMi & Summation by using current $\mathrm{i}$ or $\mathrm{s}$ indexes $-\mathrm{s}, \mathrm{i}=1,2, \ldots$ & \\
\hline $\mathrm{R}$ & Fixed groups of the ion exchangers (resins) & dimensionless \\
\hline$\Phi$ & potential of the electric field & dimensionless \\
\hline IEx & Ion Exchange, n-component IEx system & \\
\hline $1(\mathrm{~A}), 2(\mathrm{~B}), 3(\mathrm{C})$ & ions, which consist the i-concentration waves in the IEx matrix & \\
\hline $\mathrm{W}+$ & The key concept of the "i-concentration waves" for the multi-component mass transfer & \\
\hline Tfin & The completion moment $\mathrm{T}=\mathrm{T}$ fin , when the kinetic curve $\mathrm{Fi}(\mathrm{T})$ comes to the stationary plato. & dimensionless \\
\hline
\end{tabular}

[4] A. Kalinitchev, New Model of multicomponent Mass Transfer in bi-functional Matrix of Nanocomposites and results of Modelling. Protection of Metals and Physical Chemistry of Surfaces. 49: N6 (2013). in print. (in Russian \&English: 15pgs.).

[1] A. Kalinitchev, New Kinetic Computerized Model for Multicomponent Mass Transfer in Bi-functional Matrix of NanoComposites. V.2. N2, May 2013. Advances in Nanoparticles. "ANP" Edition.14pgs. Site: www. scirp.org/journal/anp.

[2] A. Kalinitchev,"Multicomponent Mass Transfer Kinetics in the Model of Nano - Composites with Bi-functional Matrix", "Smart Nano Composites" J.,"Nova" Sci. Publish. (18pgs.). (Engl.).N2.2013.Site:www.novapublhers.com/catalog/produ ct_info.php?products_id $=40111$

[3] A. Kalinitchev, "Mass Transfer Kinetics Modelling in bi-functional Ion Exchangers with Chemical Reactions on Active Centres" "IEX 2012" International IEX Conference. SCI. Ed. M. Cox. 19-21 Sept. 2012. Cambridge.(Ext. Abstr. p. 1-2).

[5] Helfferich F., Ion Exchange. Mc.Graw- Hill.NY.Ch.6. (1962). (Ionen Austauscher, Verlag Chemie, GMBH, (1959), Deutsch).

[6] Helfferich F., Ion Exchange Kinetics - Evolution of a Theory. in: "Mass Transfer \& Kinetics of IEx" (ed: L.Liberti\&F.Helfferich) M.Sijthoff \& Nordhoff. The Hague. 157-179 (1983).

[7] Petruzelli D., Helfferich F., Liberti L., Millar J., and Passino $\mathrm{R}$, Kinetics of IEx with intraparticle rate control: Models Accounting for Interaction in the solid phase. Reactive Polymers, 7, 1-13 (1987).

[8] Yoshida H., Kataoka T., Intraparticle Ion Exchange Mass Transfer in Ternary System. Kinetics. Chem. Eng. J. (1987) V.39, P.55. 
[9] Kalinitchev A., Investigation of Intraparticle IEx Kinetics in Selective Systems, in (eds.: Ja. Marinsky \& Y.Marcus) IEx \& Solv. Extr. V. 12, M.Dekker, Ch.4: 149-196 (1995).

[10] Dolgonosov A., Khamisov R., Krachak A. and Prudkovskiy A., Macroscopic model for multispecies IEx kinetics. React. Func. Polymers. 28:13-20 (1995).

[11] Dolgonosov A., Electric Effects in multicomponent IEx kinetics. React. Func. Polymers. 34: 47-51 (1999).

[12] Helfferich F. and Klein G., Multicomponent Chromatography. Theory of Interference, New York: M. Dekker Inc, (1970) $360 \mathrm{P}$.

[13] Industrial \& Engineering Chemistry Research. Journ. (prof. F. Helfferich Festschrift), Am. Chem. Soc. J. 34: 2551-2922 (1995).
[14] Tondeur D., Paradigms and Paradoxes in Modeling Adsorption and Chromatographic Separations. Ind. \& Eng. Chem. Res., 34: 2782-2788 (1995).

[15] Percolation Process: Theory and Applications, ed.: Rodrigues, A.E., Tondeur, D., The Hague: Sijthoff and Nordhoff, 1981.

[16] Kalinitchev A., Nonlinear Theory of multicomponent sorption dynamics and Chromatography. Rus. Chem. Reviews 65: 95-115 (1996).

[17] Kalinitchev A. and Hoell W., Theoretical Principles of multicomponent frontal and displacement elution Chromatography on the basis of surface complexation theory, in IEX 2008, Recent Advances in IEx Theory\&Practice, ed: M. Cox, Soc. Chem. Ind., London, 85-93 (2008). 